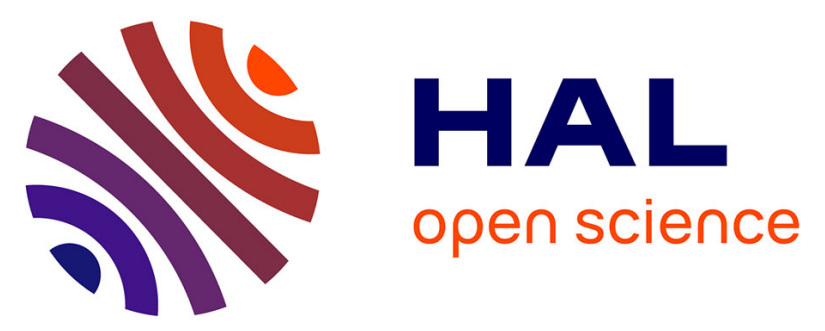

\title{
Nanosheet size-dependent rheology, microstructure, adsorption properties of graphene oxide-electrolyte dispersions and adsorbents
}

Abhijeet Ojha, Helen Ibrahim, Natalia Alyabyeva, Remi Lazzari, Michel Goldmann, Prachi Thareja

\section{To cite this version:}

Abhijeet Ojha, Helen Ibrahim, Natalia Alyabyeva, Remi Lazzari, Michel Goldmann, et al.. Nanosheet size-dependent rheology, microstructure, adsorption properties of graphene oxide-electrolyte dispersions and adsorbents. Synthetic Metals, 2020, 269, pp.116494. 10.1016/j.synthmet.2020.116494. hal-02995172

\section{HAL Id: hal-02995172 \\ https://hal.science/hal-02995172}

Submitted on 18 Nov 2020

HAL is a multi-disciplinary open access archive for the deposit and dissemination of scientific research documents, whether they are published or not. The documents may come from teaching and research institutions in France or abroad, or from public or private research centers.
L'archive ouverte pluridisciplinaire HAL, est destinée au dépôt et à la diffusion de documents scientifiques de niveau recherche, publiés ou non, émanant des établissements d'enseignement et de recherche français ou étrangers, des laboratoires publics ou privés. 


\title{
Nanosheet size-dependent rheology, microstructure, adsorption properties of graphene oxide-electrolyte dispersions and adsorbents
}

\author{
Abhijeet Ojha ${ }^{1}$, Helen Ibrahim ${ }^{2,3}$, Natalia Alyabyeva ${ }^{2}$, Rémi Lazzari ${ }^{2}$, Michel Goldmann ${ }^{2,4}$ \\ and Prachi Thareja ${ }^{5^{*}}$ \\ ${ }^{1}$ Biological Engineering, Indian Institute of Technology, Gandhinagar, Gujarat, India - 382355 \\ ${ }^{2}$ Sorbonne Université, CNRS-UMR 7588, Institut des NanoSciences de Paris, 75005, Paris \\ ${ }^{3}$ LICORNE, ECE Paris Ecole d'Ingénieurs, Immeuble POLLUX, Paris, France -75015 \\ ${ }^{4}$ Synchrotron SOLEIL L'Orme des Merisiers, Saint Aubin, Gif-sur-Yvette, France-91192 \\ ${ }^{5}$ Chemical Engineering, Indian Institute of Technology, Gandhinagar, Gujarat, India- 382355
}

\begin{abstract}
The variation of lateral dimensions of GO nanosheets by subjecting them to different ultrasonication time $(\mathrm{t}=30,60$, and $120 \mathrm{~min})$ influences the rheology, microstructure, and adsorption capacity of graphene oxide (GO) suspensions. The average storage modulus $\left(\mathrm{G}_{\mathrm{p}}^{\prime}\right)$ of GO aqueous dispersions (volume fraction $\left(\phi_{\mathrm{GO}}\right)=0.018$ ) varies as $\mathrm{GO}-30<\mathrm{GO}-60<\mathrm{GO}$ 120 , although the $\mathrm{C} / \mathrm{O}$ of $\mathrm{GO}$ nanosheets does not change significantly with ultrasonication. Addition of $10^{-5} \mathrm{M}-10^{-1} \mathrm{M} \mathrm{NaCl}$ and $\mathrm{MgCl}_{2}$ lead to a rise in $\mathrm{G}_{\mathrm{p}}^{\prime}$ as compared to electrolytefree suspensions with the formation of liquid-like, fragile gels and solid gel-like samples. Independent of time of ultrasonication, solid gels are formed at $10^{-1} \mathrm{M}$ of electrolytes. The maximum $\mathrm{G}_{\mathrm{p}}^{\prime}$ is observed in gels of GO-30 with $10^{-1} \mathrm{M} \mathrm{MgCl}_{2}$. We hypothesize that an increase in ultrasonication time causes the formation of new nanosheets with unfunctionalized edges. While $\mathrm{Na}^{+}$screens the negative charge of $\mathrm{GO}$ causing aggregation, but $\mathrm{Mg}^{2+}$ not only screens but also interacts with carboxyl groups of GO nanosheets. Therefore, the degree of $\mathrm{Mg}^{2+}$ bridging the GO nanosheets is the lowest in GO-120 and the highest in GO-30 suspensions. The adsorption capacity of methylene blue (MB) on GO-120-electrolyte lyophilized gels is the smallest, in agreement with the weak cross-linking and mechanical strength of these gels.
\end{abstract}

Keywords: Rheology, Microstructure, Graphene Oxide, Gel, Adsorption 


\section{Introduction}

Graphene, a two-dimensional material with a single atomic layer of $\mathrm{sp}^{2}$ hybridized carbon and honeycomb lattice pattern, has shown tremendous potential in electronics, composites, supracapacitors, and sensors applications owing to its exceptional optical, mechanical and thermal properties [1],[2],[3]. Interestingly, the size of the graphene sheet is found to be one of the major factors influencing its properties and potential applications, for e.g., larger graphene sheets $\left(\sim 10000 \mu \mathrm{m}^{2}\right)$ are used to construct optoelectronic devices and three-dimensional networks while graphene sheets of few nanometer sizes are used for sensing and drug delivery applications [4],[5]. It has been established that the graphene's thermal conductivity increases with increasing graphene flake's linear dimensions and eventually surpasses the thermal conductivity associated with basal planes of graphite (bulk) if the size of the flake lies in the order of few microns [6],[7]. Large-sized graphene in high yields can be synthesized by mechanical exfoliation, epitaxial growth, or by chemical vapor deposition (CVD) methods [8,9],[10]. To avoid the necessity of typical temperature procedures, expensive experimental setup and substrates during the CVD process, inorganic salts assisted direct and electrochemical assisted exfoliation methodologies are also reported in the literature. For example, Niu et al have prepared the aqueous dispersion of graphite in the presence of inorganic salts $\mathrm{NaCl}$ and $\mathrm{CuCl}_{2}$ first and subsequently re-dispersed the mixture into the orthogonal organic solvents after drying [11]. This suspension when exposed to mild ultrasonication gave rise to a few-layered large-sized $(\sim 30 \mu \mathrm{m})$. In line with this work, Parvez et al. have synthesized the large-sized $(\sim 4 \mu \mathrm{m})$, few-layered, and less oxidized graphene sheets by the electrochemical exfoliation in the aqueous dispersion of inorganic salts such as $\left(\mathrm{NH}_{4}\right)_{2} \mathrm{SO}_{4}, \mathrm{Na}_{2} \mathrm{SO}_{4}, \mathrm{~K}_{2} \mathrm{SO}_{4}$ [12]. In both the methods, the cation intercalation plays a significant role in expanding the graphite layers that lead to its exfoliation into fewlayered graphene.

Graphene oxide (GO) is a two-dimensional oxygen-rich derivative of graphene with nanoscale thickness, up to micron-scale lateral dimensions, and plenty of oxygen-containing functional groups on its conjugated planes and along the edges [13,14]. The size of the synthesized GO nanosheets can be tuned by different methods such as chemical treatment of graphite, varying the solution $\mathrm{pH}$, oxidation conditions, and optimizing the centrifugation conditions [15],[16],[17,18],[8]. Due to the high surface area and surface functionalities the GO nanosheets show excellent adsorption efficiency for complex organic dyes as well as 
capable of degrading them while using with semiconductor materials as a composite photocatalyst[19]. However due to post adsorption sedimentation in water resources, it is quite challenging to recollect them to avoid any potential secondary pollution and potential hazard to human body and aquatic environment [20]. The self-supporting three-dimensional GO-based complex architectures such as aerogels, biopolymers, and synthetic polymers cross-linked gels have demonstrated their merit over adsorbents such as carbon nanotubes (CNTs) and fullerene due to their low cost and excellent chemical and mechanical stability $[21],[22]$

Shen et al. show that GO aerogels composed of large sheets of around $30 \mu \mathrm{m}$ exhibit the fastest adsorption rate for the cationic dyes [23]. More recently, GO, and GO-based composites have been demonstrated to exhibit antibacterial properties as well. Perreault et al. show that GO-based coatings with smaller GO nanosheets have a higher antimicrobial activity than coatings composed of larger GO nanosheets [24]. The effect of different sized GO nanosheets on the performance of composite proton exchange membrane (PEM) for direct methanol fuel cells (DMFCs) has been investigated earlier and the smallest sized GO sheets have shown maximum efficiency resulting to their increased oxy-content and thus their ability to form strong hydrogen bonds [25]. The change in the lateral dimensions is also a potential way to tune the aspect ratio of GO nanosheets, which has been chosen by several research groups as an essential factor to vary and fine-tune different properties of the GO particulate suspensions. GO nanosheets form liquid crystals at a critical aspect ratio in their aqueous dispersions [26-28]. Therefore, the change in an aspect ratio of GO can influence the flow properties and, thus, the rheology of the GO aqueous suspensions. In our previous work, we have already shown that by introducing monovalent and multivalent cations, it is possible to achieve gel-like solids in the aqueous dispersion of GO with volume fraction $\left(\phi_{\mathrm{GO}}=0.018\right)[29]$.

In this study, we investigate how the lateral dimensions of the GO nanosheets influence the formation of GO-based gel-like solids, which can be utilized efficiently for water remediation purposes. We show that by introducing electrolytes with monovalent and divalent cations in the aqueous dispersions of GO with volume fraction $\phi_{\mathrm{GO}}=0.018$, solid gellike samples can be synthesized. The gel strength is dependent on the concentration, valence of the cations and the lateral dimensions of GO nanosheets. These gels are adsorbents for the cationic dye methylene blue (MB), and their adsorption capacity correlates to the gel strength. We believe our findings can be utilized to choose optimum lateral dimensions of 
GO nanosheets, concentration, and the nature of electrolytes to modulate the rheology of GO suspensions and their subsequent use as adsorbents.

\section{Experimental Section}

\subsection{Materials}

Graphite powder $\left(100 \mu \mathrm{m}\right.$ particle size), sodium nitrate powder $\left(\mathrm{NaNO}_{3}\right)$, concentrated sulfuric acid $\left(\mathrm{H}_{2} \mathrm{SO}_{4}, 98 \%\right)$ and hydrogen peroxide $\left(\mathrm{H}_{2} \mathrm{O}_{2}\right)$ are procured from SD fine chemicals. Potassium permanganate $\left(\mathrm{KMnO}_{4}\right)$ and hydrochloric acid $(\mathrm{HCl})$ are purchased from Merck Chemicals while the electrolytes magnesium chloride hex-hydrate $\left(\mathrm{MgCl}_{2} \cdot 6 \mathrm{H}_{2} \mathrm{O}\right)$, sodium chloride $(\mathrm{NaCl})$, and dye Methylene Blue $(\mathrm{MB})$ are obtained from Sigma-Aldrich. All the chemicals are used as received without any additional purification process.

\subsection{Methods}

\section{Ultrasonication assisted synthesis of GO nanosheets of variable lateral dimensions}

Hummer's process is used to synthesize GO nanosheets [16], and their lateral dimensions are varied by ultrasonication assisted size reduction process. The detailed procedure of GO synthesis is given in section $\mathrm{S}_{1}(\mathrm{a})$ of supporting information.

To synthesize the aqueous GO aqueous dispersion with the variable dimension of nanosheets, the as-prepared GO (GO-0) suspension is split into three batches of $60 \mathrm{ml}$ each and having GO concentration around $15 \mathrm{mg} / \mathrm{ml}$. Each batch is subjected to an ultrasonication (Sonic Vibracell-VC-505, $500 \mathrm{~W}, 20 \mathrm{kHz}$, amplitude 35\%, $10 \mathrm{sec}$. on/off pulse) for $30 \mathrm{~min}$., $60 \mathrm{~min}$. and $120 \mathrm{~min}$, respectively. Further, by assuming that the sound power is homogeneously distributed in the GO aqueous solution the ultra-sonic energy transferred is obtained as $193.51 \mathrm{Jg}^{-1}, 387.02 \mathrm{Jg}^{-1}$ and $774.04 \mathrm{Jg}^{-1}$ during the ultrasonication assisted synthesis of GO-30, GO-60 and GO-120 respectively. To avoid excessive overheating, the ultrasonication process is performed by surrounding the suspension with an ice bath. Aqueous dispersions are lyophilized to obtain GO nanosheets of different sizes, and the samples are tagged as GO-0 (as-prepared from Hummer's process), GO-30, GO-60, and GO120 , corresponding to the time of ultrasonication. 


\section{Preparation of different GO suspensions}

Electrolyte-free GO suspensions: $25 \mathrm{mg}\left(\phi_{\mathrm{GO}}=0.018\right)$ of lyophilized GO (GO-0, GO-30, GO-60, GO-120) are taken into $5 \mathrm{ml}$ glass vials, and $1 \mathrm{ml}$ of DI water is added to the vials followed by 5 minutes vortexing and 15 minutes of bath sonication respectively. Now, these suspensions are kept overnight at room temperature. The GO volume fraction is calculated by the formula mentioned in section $S_{1}($ b) of supporting information.

GO-electrolyte suspensions: $\mathrm{NaCl}$ and $\mathrm{MgCl}_{2}$ of varying concentrations $\left(10^{-5} \mathrm{M}-10^{-1} \mathrm{M}\right)$ are dissolved in DI water followed by the addition of $25 \mathrm{mg} / \mathrm{ml}\left(\phi_{\mathrm{GO}}=0.018\right)$ lyophilized GO (GO-0, GO-30, GO-60, GO-120) and are kept overnight at room temperature.

\subsection{Characterization}

SEM: The external morphology of the synthesized GO-0, GO-30, GO-60, and GO-120 is studied by FE-SEM micrographs obtained (with JEOL_JSM 7600F model) at operating voltages between $2-10 \mathrm{kV}$. To prepare the samples for FE-SEM analysis, around $0.5 \mathrm{mg} / \mathrm{ml}$ of GO-30, GO-60, and GO-120 aqueous dispersions are deposited on clean silicon wafers using spin coating (2000 rpm, 30 seconds). The samples are dried before the analysis, and a platinum coating of few nanometers is deposited on the sample surfaces to avoid charging. However, GO-electrolyte suspensions are lyophilized and are then sprinkled on the carbon tape in a minuscule quantity. These samples are imaged as it is after the platinum coating.

AFM: The height profile and lateral dimensions of synthesized GO-30, GO-60, and GO-120 nanosheets are obtained with Atomic force microscopy (Bruker, AFM, Multimode 8.0). The height and phase images are obtained with tapping mode at a scan rate of $1.5 \mathrm{~Hz} .0 .3 \mathrm{mg} / \mathrm{ml}$ aqueous dispersions of GO-30, GO-60, and GO-120 are spin-coated (2000 rpm, 30 seconds) on a cleaned silicon wafer. The samples are then dried in a vacuum desiccator for $\sim 2$ days. The images are processed with the Nanoscope analysis software. A total of $60 \mathrm{GO}$ nanosheets are measured at various locations to calculate the average lateral dimensions and height of the nanosheets. 
FTIR: The spectra of GO nanosheets synthesized with different ultrasonication times and the corresponding GO-electrolyte suspensions are recorded with FTIR spectrometer (Perkin

Elmer, Spectrum 2) using ATR mode in the wave numbers range of $500-4000 \mathrm{~cm}^{-1}$. A minute quantity of each sample is positioned on the sample stage and fixed using the vertical knob. To effectively avoid the moisture content, the samples are placed inside the vacuum desiccator before the analysis.

XPS: The XPS, (SPECS, Germany) is performed using focused monochromatic Al ka lines $\left(h v=1486.6 \mathrm{eV}\right.$, pass energy of $E_{p}=10 \mathrm{eV}$ ) to quantify the ratio between carbon and oxygen elements. XPS spectra are also used to understand any potential modification of functional groups on GO sheets with ultrasonication time. Before the analysis, GO samples are deposited on gold coated silicon wafer and dried in a desiccator to get-rid of surface contaminants. The background of spectra is removed using spline function and the baseline is fixed. The curve fitting is performed by Levenberg-Marquardt (L-M) method and the peaks are represented by Gaussian line shape and all the bound constraints are limited to the simple variable domain associated with L-M method. The $\mathrm{R}^{2}$ values are found to be in the range of 0.98-0.99 for all the fittings and the $\mathrm{sp}^{2}$ carbon peak at $284.6 \mathrm{eV}$ is chosen as an internal reference.

\subsection{Rheology}

Anton Paar modular compact rheometer (MCR 302) with stress and strain controlled capability is used for the rheological analysis of aqueous suspensions of GO-0, GO-30, GO60, GO-120, and the respective GO-electrolyte suspensions. At a constant temperature of $20^{\circ} \mathrm{C}$, rheological experiments are performed using a cone and plate tool with $25 \mathrm{~mm}$ diameter and a truncation angle of $2^{\circ}$. A constant gap of $0.105 \mathrm{~mm}$ is maintained between the geometry and the Peltier, and a solvent trap is used to avoid the solvent evaporation. In literature also, research groups have tested the rheological properties of GO aqueous dispersions at quite lower volume fractions than this study using the cone and plate geometry and did not notice any leakage of sample at higher angular frequencies $(\omega)$. For example, Konkena et al .[30], Naficy et al. [31] have studied very low $\phi_{\mathrm{GO}}$ as 0.0013 to 0.022 with or without changing the ionic conditions using the same cone and plate geometry and similar gap settings. Oscillatory time sweep experiment is performed on a constant angular 
frequency $(\omega)$ of $6.28 \mathrm{rad} / \mathrm{s}$. Further, to understand the viscoelastic behavior, SAOS (Small Amplitude Oscillatory Frequency Sweep) measurements are performed. The samples are subjected to a variable angular frequency $(\omega)$ in the range of $0.1-100 \mathrm{rad} / \mathrm{s}$ at a constant oscillatory strain $(\gamma)=0.1 \%$ and all the measurements of storage moduli $\left(\mathrm{G}^{\prime}\right)$ and loss moduli $\left(\mathrm{G}^{\prime \prime}\right)$ are restricted to the linear viscoelastic region. Further, the non-linear response is probed by LAOS (Large Amplitude Oscillatory Strain) sweep measurements at applied oscillatory $\gamma$ $=0.01-1200 \%$ and at a constant $\omega=6.28 \mathrm{rad} / \mathrm{s}$.

\subsection{Dye Adsorption Experiments}

$5 \mathrm{mg}$ of GO-30, GO-60, GO-120, and the respective GO-electrolyte suspensions are taken in lyophilized form in glass vials filled with Methylene Blue (MB) (Mol. weight $319.65 \mathrm{~g} \mathrm{~mol}^{-}$

${ }^{1}$ ). The concentration of MB is $20 \mathrm{ppm}$ in DI water. The vials are covered properly and then kept on a constantly shaking platform. The concentration of MB dye remaining in the solution after adsorption is estimated by the absorbance measurement at $665 \mathrm{~nm}$ and using the standard MB calibration curve represented as section $S_{2}$ of supporting information.

\subsection{Quantification of electrolyte leaching using ICP-OES studies}

The quantification of leached electrolytes to water from the GO-electrolyte gels is performed by ICP-OES studies using Perkin Elmer Avio 200 optical emission spectrometer. Lyophilized GO-electrolyte gels $(5 \mathrm{mg}$ ) are immersed in glass vials filled with $20 \mathrm{ml}$ of DI water. The aliquots are collected at $\mathrm{t}=0,24$, and $60 \mathrm{hrs}$. respectively. All the aliquots are then diluted by $2 \% \mathrm{HNO}_{3}$ before further studies.

\section{Results and Discussion}

\subsection{Characterization of synthesized GO nanosheets}

The morphology of synthesized GO-30, GO-60 and GO-120 nanosheets is observed under FE-SEM. The images show GO sheets with circular ring-like structures looking smooth at the surfaces while wrinkled at the edges (Fig. 1). It can also be inferred from the FE-SEM micrographs that the ultrasonication is efficiently able to reduce the lateral dimensions of GO nanosheets. 

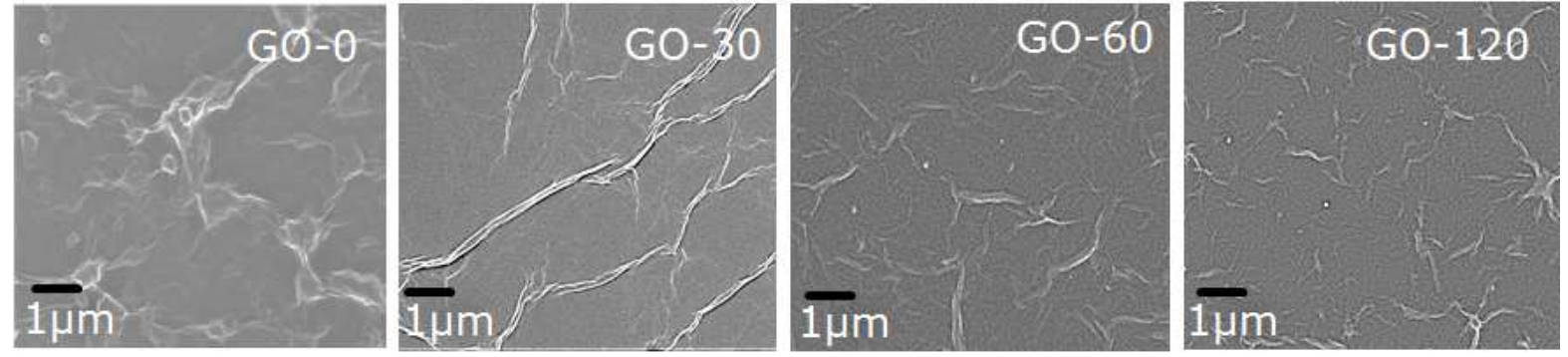

Fig. 1 FE-SEM micrographs of as prepared GO nanosheets (GO-0) and GO nanosheets after ultrasonication for 30, 60 and 120 minutes respectively (GO-30, GO-60 and GO-120)

The height profile measured from AFM reveals the GO nanosheet thickness as approximately 1.4-1.7 nm (Fig. 2 (a)-(f)). The distribution of lateral dimensions of GO nanosheets is shown in Fig. 2 (g)-(i). It can be inferred from the size histogram that for GO30 sample, most of the GO sheets (>50\%) lie within the size range of 450-600 $\mathrm{nm}$ with the average lateral size of $470 \mathrm{~nm}$. Similarly, for GO-60 almost $60-70 \%$ of nanosheets are in size range of 300-450 $\mathrm{nm}$ with an average lateral dimension of $\sim 368 \mathrm{~nm}$. For GO-120 samples, the sheets are less than $300 \mathrm{~nm}$ with an average lateral dimension of $\sim 260 \mathrm{~nm}$. Thus by considering the thickness of GO nanosheets as $1.4 \mathrm{~nm}$, the aspect ratios of GO-30, GO-60, and GO-120 are calculated as 336, 262, and 185, respectively. 

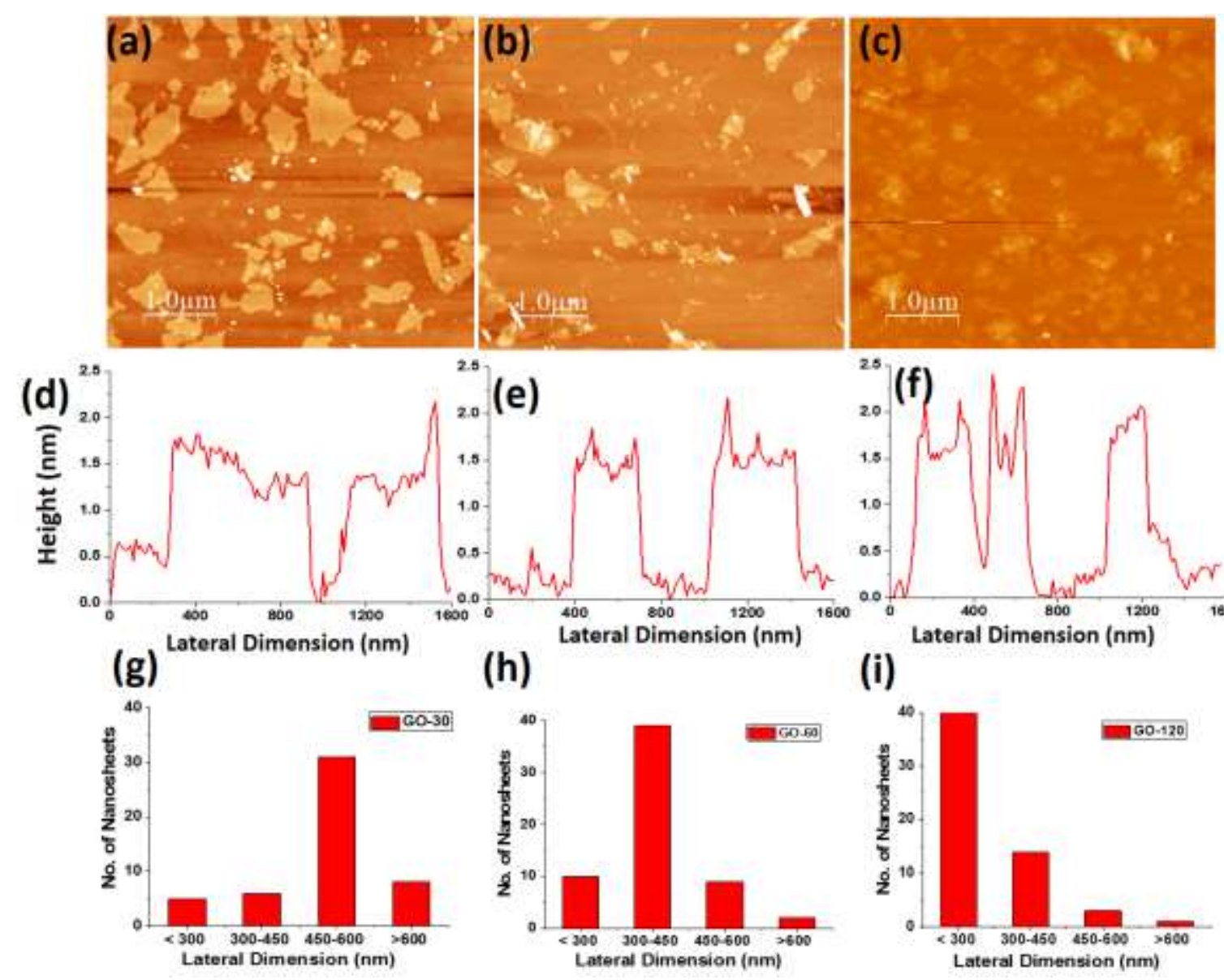

(e)

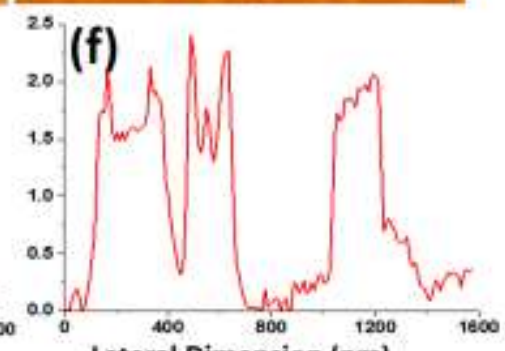

(h)

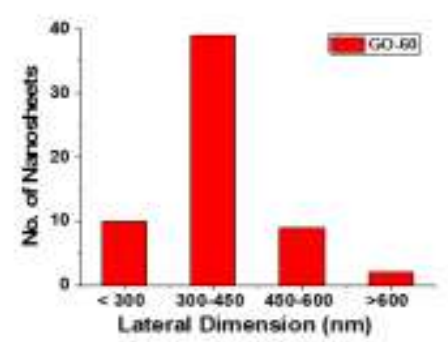

(i)

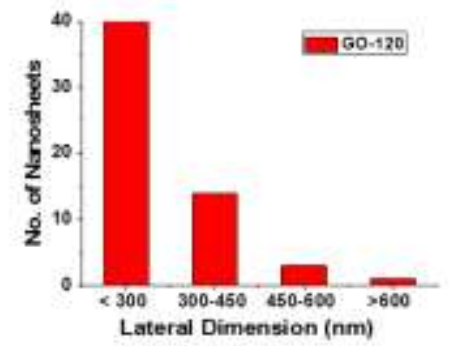

Fig. 2. Tapping mode two-dimensional images of GO nanosheets deposited on a silicon wafer with corresponding height profiles below each image (a, d) GO-30 (b, e) GO-60 and (c, f) GO-120. Size (lateral dimension) histogram for (g) GO-30, (h) GO-60, (i) GO-120 nanosheets

The FTIR spectroscopy studies the chemical structure and the surface functional groups present on the GO nanosheets. The FTIR spectra obtained for GO-0 (as prepared), GO-30, GO-60, and GO-120 are shown in Fig. 3. In the FTIR spectrum, the broad and intense peak around $3350 \mathrm{~cm}^{-1}$ is associated with $-\mathrm{OH}$ vibrations as well as free and intercalated water molecules. The carboxyl group $(\mathrm{C}=\mathrm{O})$ shows a strong stretching vibration peak at $1733 \mathrm{~cm}^{-1}$. The spectrum range $1500-1000 \mathrm{~cm}^{-1}$ represents various $\mathrm{C}-\mathrm{O}$ vibrations in the form of three characteristic peaks. The first peak is situated at $1380 \mathrm{~cm}^{-1}$, which originates from the bending and deformation vibrations related to the carboxyl (C-O) group of GO. The second peak around $1287 \mathrm{~cm}^{-1}$ is related to epoxy C-O vibrations, while the third peak around $1287 \mathrm{~cm}^{-1}$ is associated with the alkoxy C-O vibrations in GO. The results indicate that the increase in the time of ultrasonication does not cause any significant change 
in the chemical structure of GO nanosheets. The FTIR spectra of GO shows good agreement with the reported literature $[32,33]$.

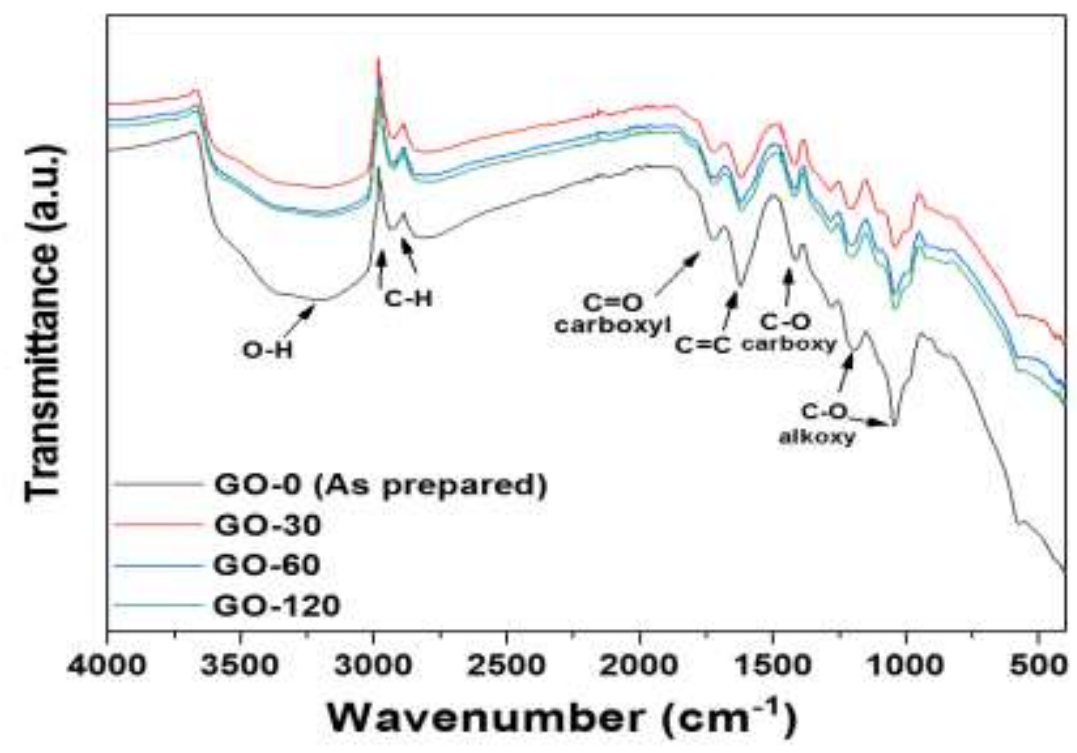

Fig. 3. FTIR spectra of GO-0, GO-30, GO-60, GO-120 nanosheets

The XPS measurements help to decipher the surface chemical composition of GO nanosheets after different ultrasonication times, and the obtained spectra are shown in Fig. 4. The XPS spectra of GO nanosheets represents the non-oxygenated or graphitic carbon rings $(\mathrm{C}=\mathrm{C}, 284.6 \mathrm{eV})$ and two significant components of oxygen-bearing groups as epoxy/hydroxyl groups (C-O, $286.7 \mathrm{eV})$ and carbonyl/carboxyl group $(\mathrm{C}=\mathrm{O}, 287.6 \mathrm{eV})$. Due to the predominant grafting of epoxy and hydroxyl functional groups on $\mathrm{sp}^{2}$ bonded graphitic carbon network rather than edges, a relatively sharper C1s XPS peak is obtained at $286.7 \mathrm{eV}$ (representing epoxy/hydroxyl functional groups) as compared to a smaller peak at $287.6 \mathrm{eV}$ (representing carbonyl/carboxyl group) [33]. It is observed that the $\mathrm{C} / \mathrm{O}$ ratio of $\mathrm{GO}$ nanosheets falls from 2.95 (GO-30) to 2.34 (GO-60) and reaches up to 2.31 (GO-120). This small change in $\mathrm{C} / \mathrm{O}$ ratio reveals no considerable variation in the carboxyl, hydroxyl, carbonyl and epoxy functional groups concentrations on GO sheets. 

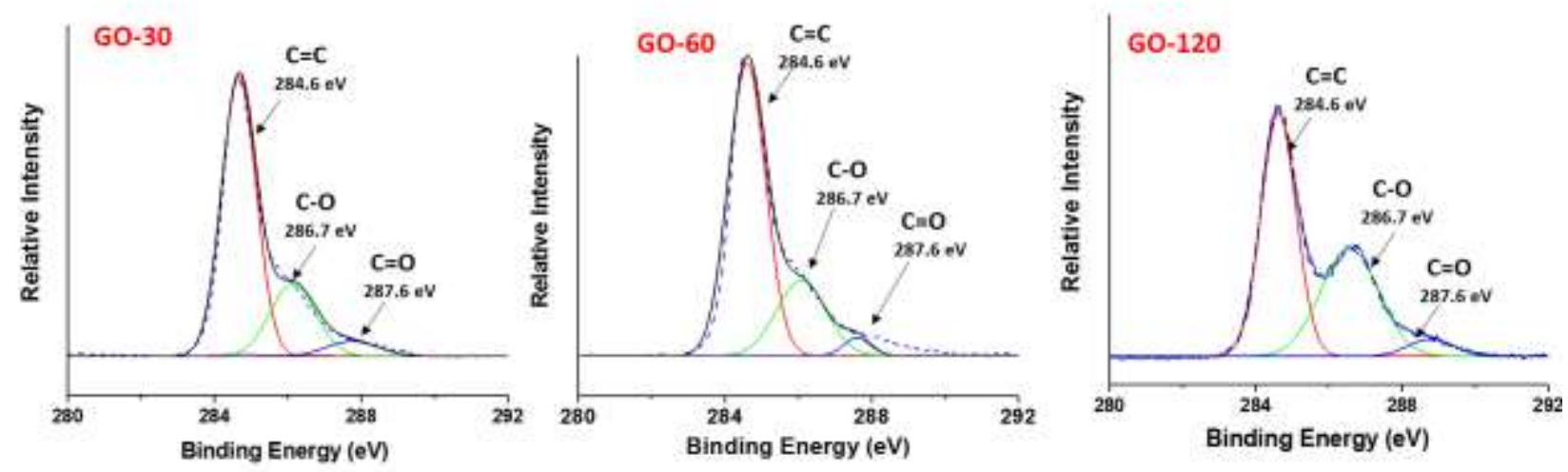

Fig. 4 Deconvoluted XPS spectrum of C1s of GO sheets after ultrasonication for 30, 60 and 120 minutes

\subsection{Rheology of ultrasonicated GO suspensions}

\section{(a) Electrolyte-free GO-30, GO-60, and GO-120 suspensions}

At $\phi_{\mathrm{GO}}=0.018$, GO-30, GO-60, and G-120 aqueous suspensions are mostly liquid-like, which flow upon tilting the sample vials. The frequency sweep data of Fig. 5 (a) shows that $\mathrm{G}^{\prime}$ for the aqueous suspensions varies in the order GO-30<GO-60 < GO-120. The data also indicates that $\mathrm{G}^{\prime}$ is always higher than $\mathrm{G}^{\prime \prime}$ and weakly dependent on $\omega$, suggesting that GO30, GO-60, and GO-120 suspensions are viscoelastic solid samples. Fig. 5 (b) represents the time average $G^{\prime}$ or $G_{p}^{\prime}$ deduced from the small amplitude oscillatory time sweep experiments (section $\mathrm{S}_{3}$ of supporting information). $\mathrm{G}_{\mathrm{p}}^{\prime}$ for $\mathrm{GO}-30$ suspensions is $46.4 \mathrm{~Pa}$, which increases to $124 \mathrm{~Pa}$ and finally to $170.5 \mathrm{~Pa}$ for GO-60 and GO-120 samples, respectively. Therefore, as the time of ultrasonication increases or the lateral dimensions of the GO nanosheets decrease, the elastic moduli of the suspensions increase. The large amplitude oscillatory strain sweep (LAOS) experiments indicate that the moduli ( $\mathrm{G}^{\prime}$ and $\left.\mathrm{G}^{\prime \prime}\right)$ of the suspensions are independent of oscillatory strain $(\gamma)$ when $\gamma<1 \%$. At $\gamma>1 \%$, G' shows a cross-over with G" and eventually G" exceeds G' indicating flow induced yielding [34],[35]. 


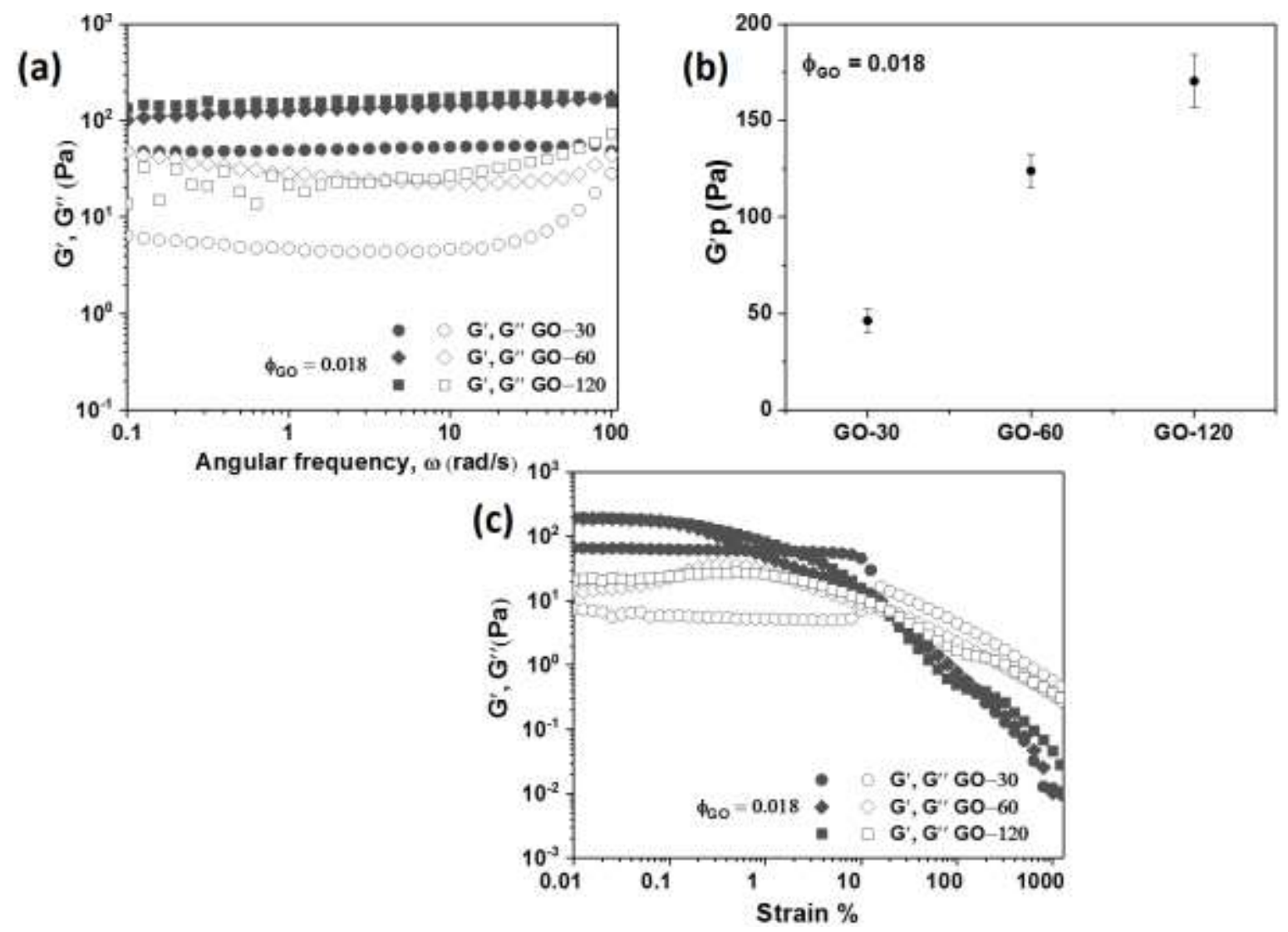

Fig. 5 Dynamic rheological behavior of aqueous GO-30, GO-60, and GO-120 suspensions at $\phi_{\mathrm{GO}}=0.018$ (a)

Dynamic frequency sweep (b) Average $\mathrm{G}_{\mathrm{p}}^{\prime}$ deduced from oscillatory time sweep experiment (c) Large amplitude oscillatory sweep experiments. Error bars show the maximum possible deviation from the average values

The zeta potential measurements show that GO-30, GO-60, and GO-120 samples have a zeta potential of $-49.1 \mathrm{mV},-47.4 \mathrm{mV}$, and $-46.4 \mathrm{mV}$ respectively. It is reported that the epoxy and hydroxyl functional groups are located at the basal plane of the GO nanosheets, and the carboxyl groups are attached to the edges [13]. The ionization of carboxyl groups in water renders GO a native negative surface charge, which is also evident by the values of zeta potential. The zeta potential values more than $-30 \mathrm{mV}$ suggest that there is a negligible loss of the oxygen functionalities from the overall GO surface considered. This is in agreement with the XPS spectra of Fig. 4. We propose that the reduction of the lateral size of the GO nanosheets due to ultrasonication also causes the formation of smaller sized GO nanosheets with newly formed edges, which are hydrophobic in nature and do not have the sufficient carboxylic acid group coverage. This causes GO aggregation and the aggregation tendency of GO nanosheets rises with increasing ultrasonication time to reduce the contact of hydrophobic edges to water. The increased aggregation of GO nanosheets 
leads to a significant increase in $\mathrm{G}_{\mathrm{p}}^{\prime}$ in particular order GO-30 < GO-60 < GO-120. Compton et al also report the formation of solid-like GO hydrogels $\left(\mathrm{G}^{\prime} \sim 1000 \mathrm{~Pa}\right)$ with $\mathrm{GO}$ nanosheets of lateral dimensions $\sim 80 \mathrm{~nm}$ formed after ultrasonicated for $120 \mathrm{~min}$ [36]. The authors propose that the ultrasonication induced aggregation leads to the formation of large GO clusters which have an uneven distribution of hydrogen bond forming oxygenated functional groups on their edges. The hydrogen bonding between the aggregates mediated by water leads to the formation of GO networks. The particulate GO networks are held by a variety of interactions including Van der Waals, hydrogen bonding between functional groups of GO and $\pi-\pi$ stacking of the $\mathrm{sp}^{2}$ regions on the GO basal planes. A similar mechanism of aggregation of GO nanosheets in the presence of water and the subsequent mechanical properties is also elucidated by the MD simulations by Medhaker et al [37]. The authors show that the structural and mechanical properties of GO paper-like samples are controlled by hydrogen bonds, interlayer and intra-layer $\mathrm{H}$-bonds between the oxygenated functional groups of GO nanosheets and H-bonds with water molecules.

\section{(b) Addition of electrolytes in GO-30, GO-60, and GO-120 suspensions}

As shown in Fig. 6 (a), visually three different types of GO-electrolyte suspensions are observed on the basis of their flow characteristics upon inverting the sample vials: liquid-like suspensions, fragile gels that flow over time and robust gels that do not flow during the observation time. Based on these visual observations, phase diagrams are prepared for GO$\mathrm{NaCl}$ and $\mathrm{GO}-\mathrm{MgCl}_{2}$ suspensions as a function of $[\mathrm{NaCl}],\left[\mathrm{MgCl}_{2}\right]$, and the ultrasonication time used to prepare GO nanosheets (Fig. 6 (b), (c)). As evident, visually liquid-like suspensions are observed for both $[\mathrm{NaCl}],\left[\mathrm{MgCl}_{2}\right]=10^{-5} \mathrm{M}$ irrespective of the time of ultrasonication of GO nanosheets except for GO-30. Also GO-120 suspension is liquid-like with $[\mathrm{NaCl}]=10^{-4} \mathrm{M}$.

GO-30 forms fragile gels when $10^{-5} \mathrm{M}[\mathrm{NaCl}]$ and $\left[\mathrm{MgCl}_{2}\right]$ are introduced into the suspension. Fragile gels are also formed for GO-30 and GO-60 with $[\mathrm{NaCl}]$ and $\left[\mathrm{MgCl}_{2}\right]=$ $10^{-4} \mathrm{M}$; GO-120 with $10^{-4} \mathrm{M}\left[\mathrm{MgCl}_{2}\right]$, GO-60, GO-120 with $\left[\mathrm{MgCl}_{2}\right]=10^{-3} \mathrm{M}$ and GO-60 with $[\mathrm{NaCl}]=10^{-3} \mathrm{M}$.

Robust solid gels are observed for GO-30 with $[\mathrm{NaCl}]$ and $\left[\mathrm{MgCl}_{2}\right]=10^{-3} \mathrm{M}, 10^{-2} \mathrm{M}$ and $10^{-1} \mathrm{M}$; GO-60 at $[\mathrm{NaCl}]=10^{-2} \mathrm{M}, 10^{-1} \mathrm{M}$; $\left[\mathrm{MgCl}_{2}\right]=10^{-3} \mathrm{M}-10^{-1} \mathrm{M}$; GO-120 with $[\mathrm{NaCl}]=10^{-2} \mathrm{M}$ and $\left[\mathrm{MgCl}_{2}\right]=10^{-1} \mathrm{M}$. 

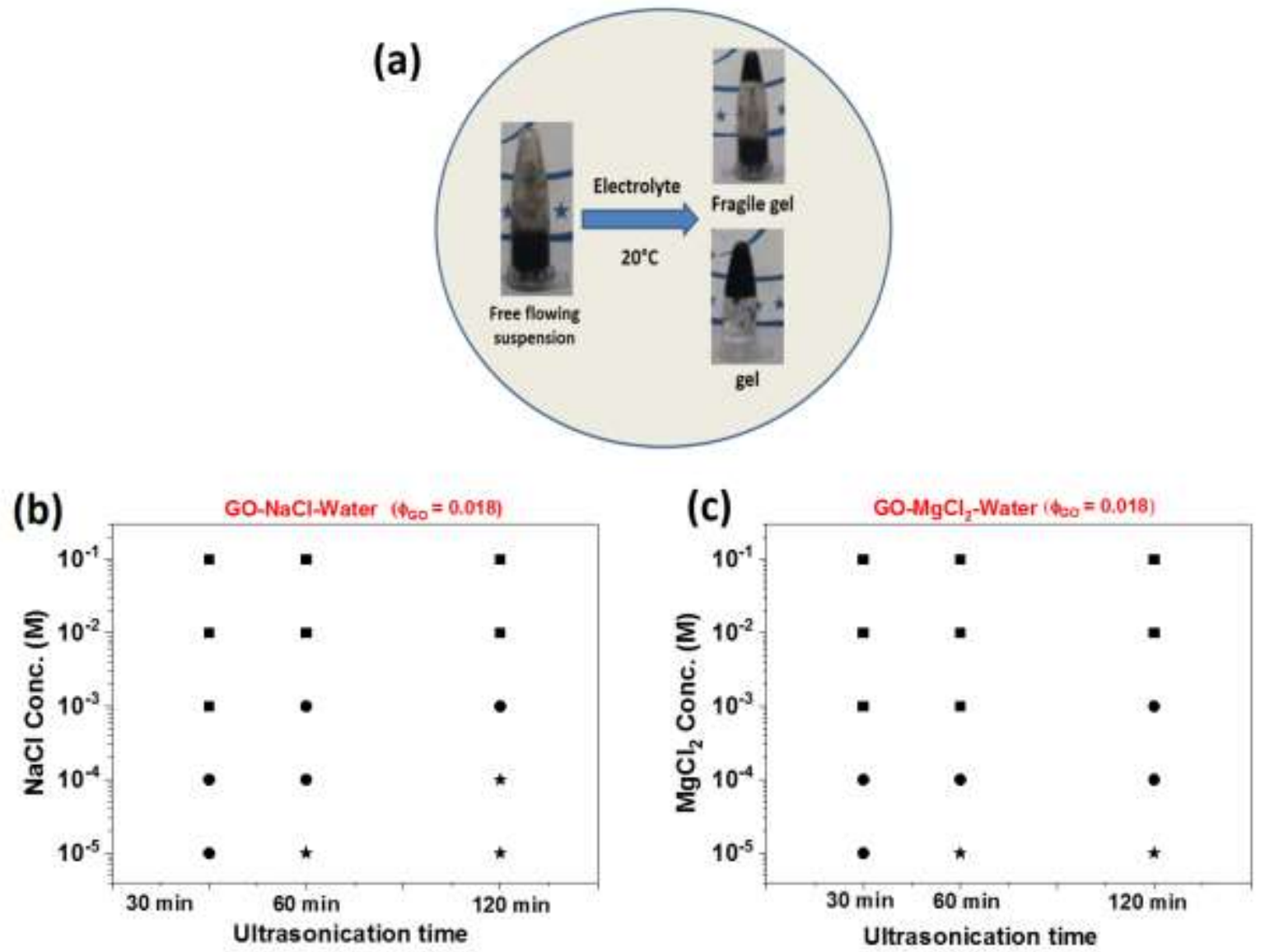

Fig. 6 Phase diagrams for GO-electrolyte suspensions ultrasonicated for 30, 60 and 120 minutes. In the phase

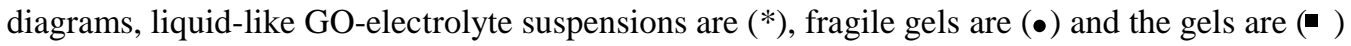

Fig.7 (a) - (f) show the frequency sweep spectrum of aqueous GO-30, GO-60, and GO-120 suspensions at $\phi_{\mathrm{GO}}=0.018$ in the presence of electrolytes $\mathrm{NaCl}$ and $\mathrm{MgCl}_{2}$. As shown in Fig. 7, visually liquid-like GO-60, GO-120 suspensions with $10^{-5} \mathrm{M}[\mathrm{NaCl}]$ and $\left[\mathrm{MgCl}_{2}\right]$ are viscoelastic solids with $\mathrm{G}^{\prime}>\mathrm{G}^{\prime \prime}$ in the tested experimental range of $\omega$ and $\mathrm{G}^{\prime}$ is weakly dependent on $\omega$. The spectra also show that there is no direct cross-over between the $\mathrm{G}^{\prime}$ and $\mathrm{G}^{\prime \prime}$ in the tested range of $\omega$, indicative of long structural relaxation times. Similar frequency sweep spectra are observed for suspensions with $10^{-3} \mathrm{M}, 10^{-1} \mathrm{M}[\mathrm{NaCl}]$ and $\left[\mathrm{MgCl}_{2}\right]$. For a better understanding of the influence of ultrasonication time and electrolytes, the average $G_{p}^{\prime}$ are extracted from small amplitude oscillatory time sweep experiments (section $\mathrm{S}_{4}(\mathrm{a}, \mathrm{b})$ of supporting information). 
(a)
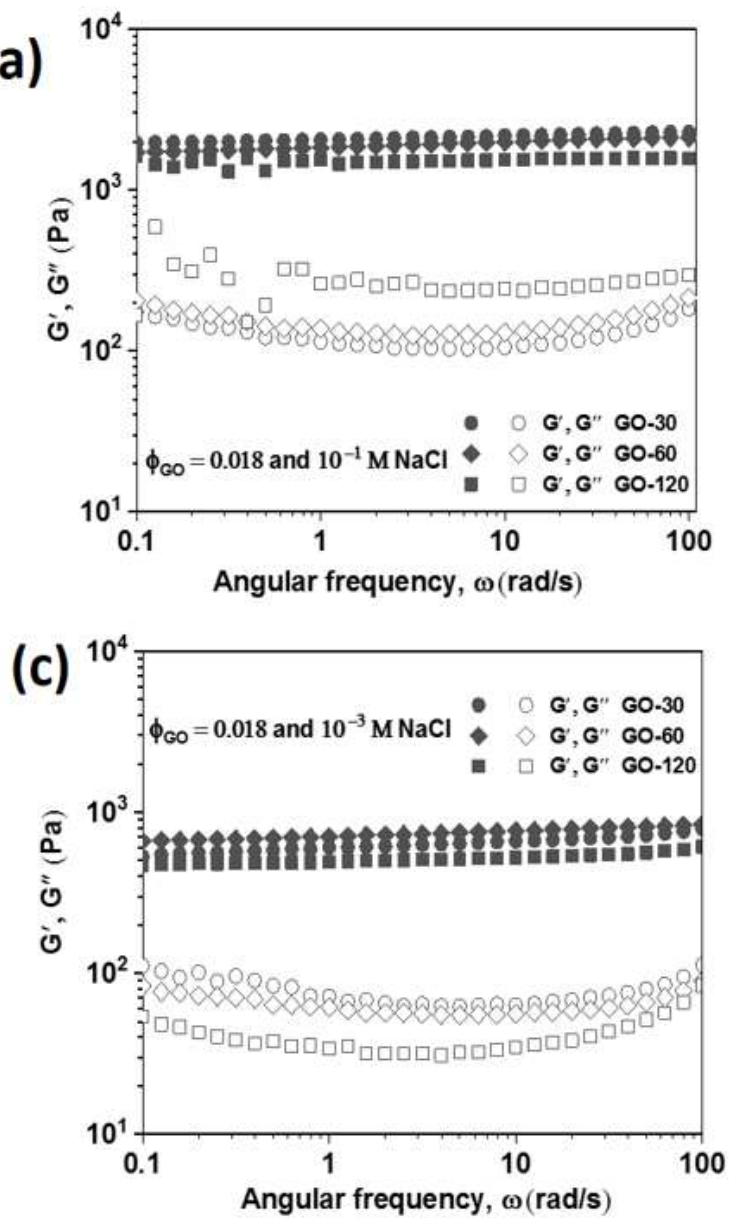

(e)

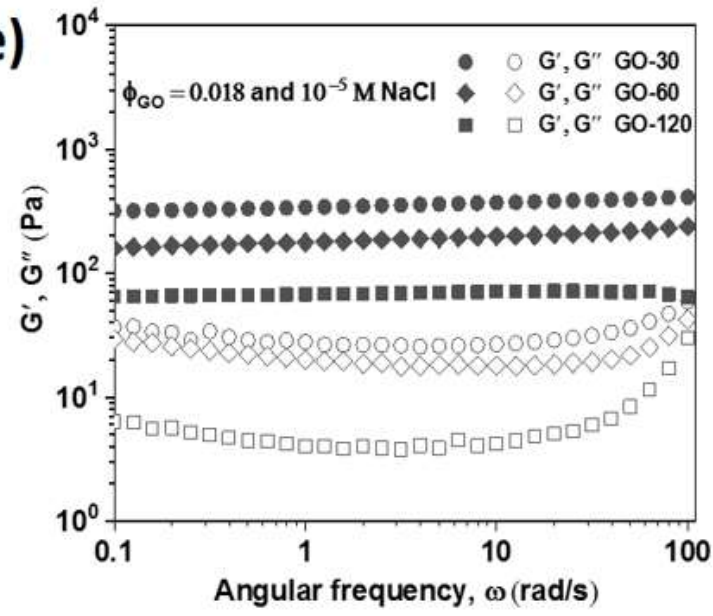

(b)

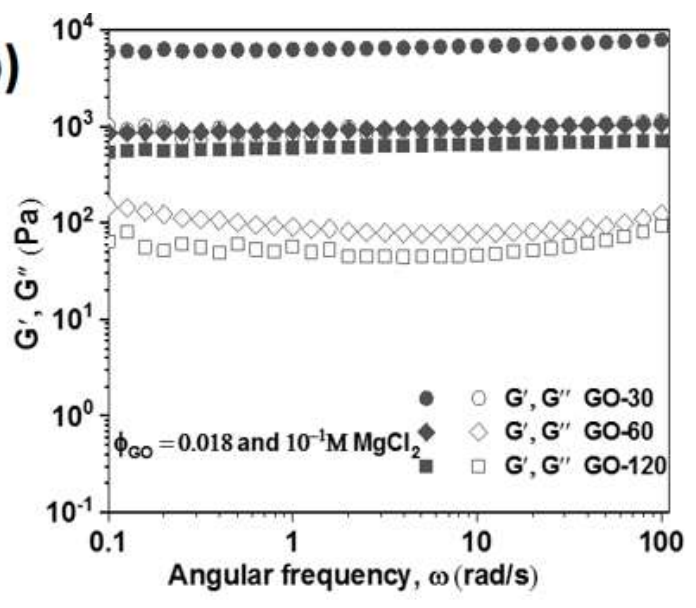

(d)

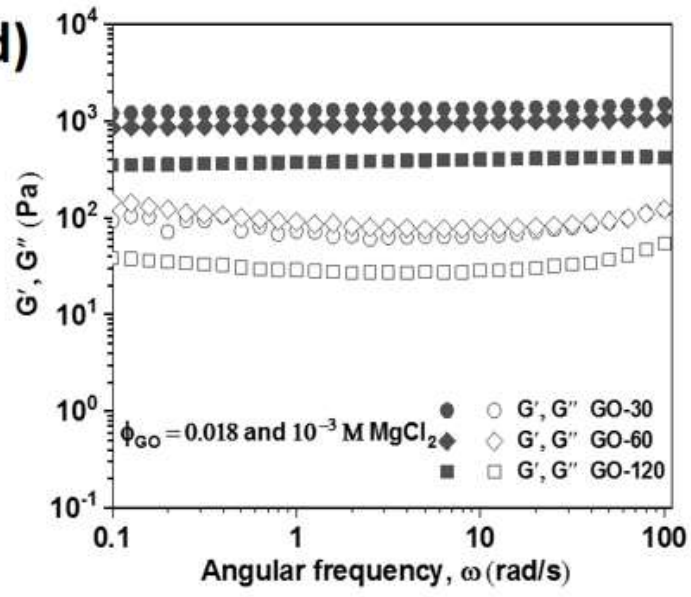

(f)

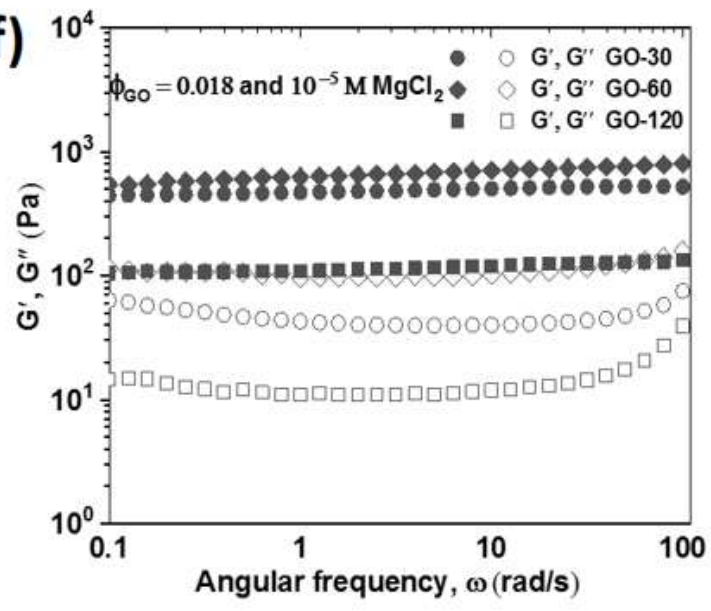

Fig. 7 Dynamic rheology of GO-electrolyte suspensions with varying (a, c, e) $[\mathrm{NaCl}]$ and (b, d, f) $\left[\mathrm{MgCl}_{2}\right]$

As shown in Fig. 8, visually liquid-like GO-60, GO-120 suspensions with $10^{-5} \mathrm{M}[\mathrm{NaCl}]$ have a $\mathrm{G}_{\mathrm{p}}^{\prime}$ of $181 \mathrm{~Pa}$ and $67 \mathrm{~Pa}$ respectively. Liquid-like GO-60, GO-120 suspensions with $10^{-5} \mathrm{M}\left[\mathrm{MgCl}_{2}\right]$ have the $\mathrm{G}_{\mathrm{p}}^{\prime}$ values $312 \mathrm{~Pa}$ and $106 \mathrm{~Pa}$ respectively, slightly higher than 
electrolyte-free suspensions. Fragile gels of GO-30 suspensions with $[\mathrm{NaCl}],\left[\mathrm{MgCl}_{2}\right]=10^{-5}$ $\mathrm{M}$ have a $\mathrm{G}_{\mathrm{p}}^{\prime}$ of $338 \mathrm{~Pa}$ and $465 \mathrm{~Pa}$ respectively. Similarly, fragile gels of GO-120 suspensions with $[\mathrm{NaCl}],\left[\mathrm{MgCl}_{2}\right]=10^{-3} \mathrm{M}$ have $\mathrm{G}_{\mathrm{p}}^{\prime}$ of $483 \mathrm{~Pa}$ and $611 \mathrm{~Pa}$ respectively.

Solid gels of GO-30, GO-60 and GO-120 suspensions with $[\mathrm{NaCl}]=10^{-1} \mathrm{M}$ have $\mathrm{G}_{\mathrm{p}}^{\prime}$ $2040 \mathrm{~Pa}, 1850 \mathrm{~Pa}$ and $1510 \mathrm{~Pa}$ respectively (Fig. 8 (a)). However, the addition of $10^{-1} \mathrm{M}$ $\left[\mathrm{MgCl}_{2}\right]$ to GO-30, GO-60, and GO-120 gives rise to the $\mathrm{G}_{\mathrm{p}}^{\prime}$ up to $6270 \mathrm{~Pa}, 2030 \mathrm{~Pa}$, and $1630 \mathrm{~Pa}$ respectively (Fig. 8 (b)). It can also be deduced from the bar charts that independent of $[\mathrm{NaCl}]$ or $\left[\mathrm{MgCl}_{2}\right]$, the $\mathrm{G}_{\mathrm{p}}^{\prime}$ for $\mathrm{GO}-\mathrm{MgCl}_{2}$ suspensions are relatively higher than $\mathrm{GO}-\mathrm{NaCl}$ suspensions. Also, at all $[\mathrm{NaCl}]$ or $\left[\mathrm{MgCl}_{2}\right], \mathrm{G}_{\mathrm{p}}^{\prime}$ decreases with the increase in time of ultrasonication. The minimum $\mathrm{G}_{\mathrm{p}}^{\prime}(1510 \mathrm{~Pa})$ is observed for gels of GO-120 suspensions with $[\mathrm{NaCl}]=10^{-1} \mathrm{M}$ and the maximum $\mathrm{G}_{\mathrm{p}}^{\prime}(6270 \mathrm{pa})$ is observed for gels of GO-30 with $\left[\mathrm{MgCl}_{2}\right]=10^{-1} \mathrm{M}$. As shown by Fig. 8(c), GO-120 based suspensions show minimum $\mathrm{G}_{\mathrm{p}}^{\prime}$ as compare to its other counterparts as GO-30 and GO-60, however the $\mathrm{G}_{\mathrm{p}}^{\prime}$ trend remains similar. Another way to show the strength of prepared gels is to represent their average $G^{\prime} / G^{\prime \prime}$ at any $\omega$ value, a colloidal gel with higher $G^{\prime} / G^{\prime \prime}$ is expected to show higher strength. Fig. 8 (d) shows the average $G^{\prime} / G^{\prime \prime}$ values for all the tested GO-electrolyte suspensions. Interestingly, all the 'gels' mentioned in phase diagram (Fig. 6), show G'/G" >10 and the visually liquid like samples have relatively less $G^{\prime} / G^{\prime \prime}$ values. These results also confirm the concomitancy of visual and experimental (rheology) observations. 

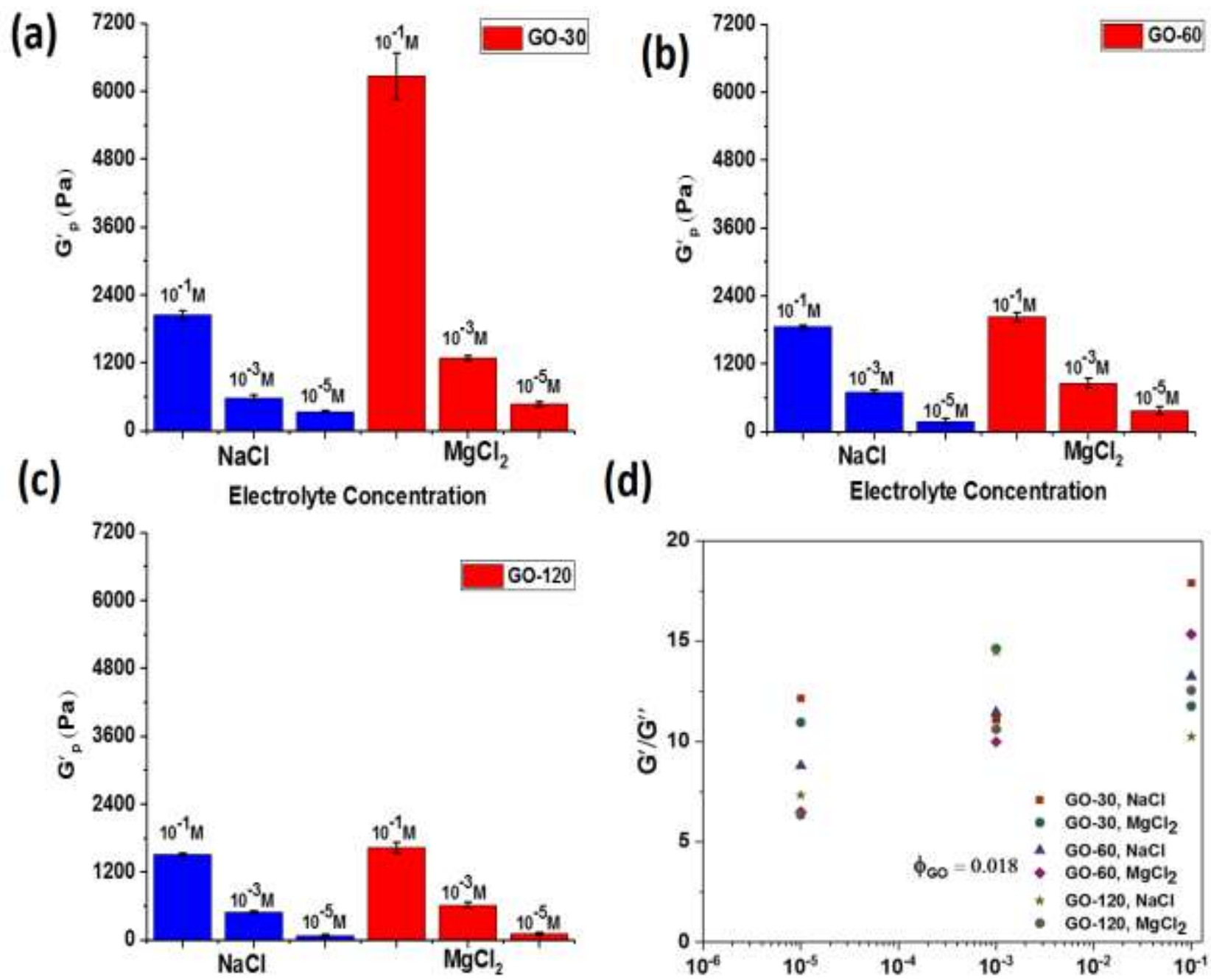

Electrolyte Concentration

(d)

Electrolyte Concentration

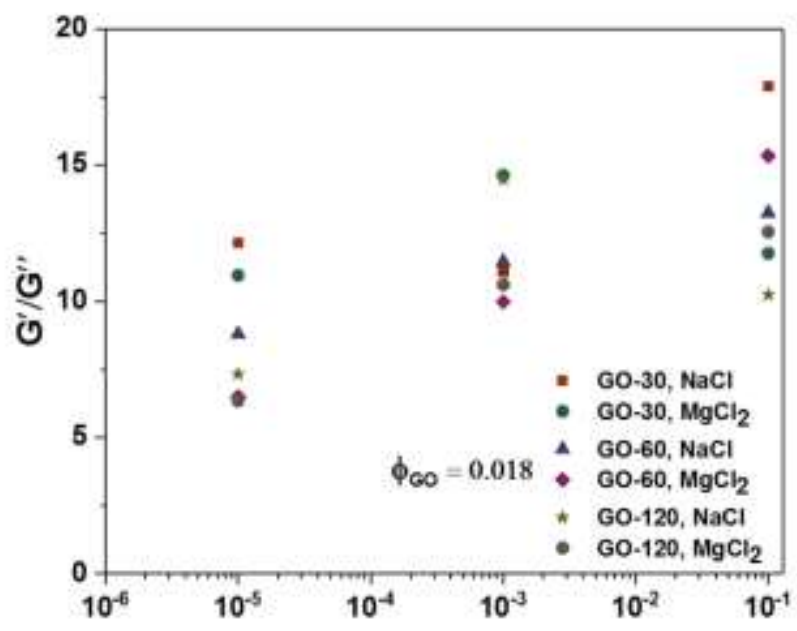

Electrolyte Concentration (M)

Fig. 8 (a) $\mathrm{G}_{\mathrm{p}}^{\prime}$ of GO-30 (b) GO-60 and (c) GO-120 with varying [NaCl] and $\left[\mathrm{MgCl}_{2}\right]$ (d) $\mathrm{G}^{\prime} / \mathrm{G}^{\prime \prime}$ for different $\mathrm{GO}$ electrolyte gels at $\omega=1 \mathrm{rad} / \mathrm{s}$. with different electrolyte concentrations as $10^{-5} \mathrm{M}, 10^{-3} \mathrm{M}$ and $10^{-1} \mathrm{M}$

The LAOS (large amplitude oscillatory strain sweep) experiments for GO-30, GO-60, GO-120 suspensions in the presence of $10^{-5} \mathrm{M}, 10^{-3} \mathrm{M}$, and $10^{-1} \mathrm{M}[\mathrm{NaCl}]$ and $\left[\mathrm{MgCl}_{2}\right]$ are presented in section $S_{5}$ of supporting information. The data indicates that $G^{\prime}>G^{\prime \prime}$ for all the tested suspensions with both the moduli ( $G^{\prime}$ and $\left.G^{\prime \prime}\right)$ being independent of applied $\gamma<0.2 \%$. Beyond $\gamma=0.2 \%, \mathrm{G}^{\prime}$ and $\mathrm{G}^{\prime \prime}$ show cross over with $\mathrm{G}^{\prime \prime}$ surpassing $\mathrm{G}$,' a behavior similar to the yielding of colloidal gels [34,38],[35].

As shown in section $\mathrm{S}_{6}$ of supporting information, two strain peaks can be observed in the $\mathrm{G}^{\prime} \gamma$ versus $\gamma \%$ graphs of GO-electrolyte suspensions which are solid gel-like samples, e.g. GO-30 with $[\mathrm{NaCl}]$ and $\left[\mathrm{MgCl}_{2}\right]=10^{-3} \mathrm{M}$ and $10^{-1} \mathrm{M}$; GO-60 with $\left[\mathrm{MgCl}_{2}\right]=10^{-3} \mathrm{M}, 10^{-1}$ 
M; GO-120 with $[\mathrm{NaCl}]$ and $\left[\mathrm{MgCl}_{2}\right]$ of $10^{-1} \mathrm{M}$. The 'two-peaks' or two 'yield-points' in the LAOS experiments (section $S_{6}$ ) of the gel-like suspensions correspond to the breaking of inter-cluster bonds between GO nanosheet flocs and the rupture of inter-particle bonds between these flocs causing fluidization of the samples [34].

\subsection{Microstructure of GO-electrolyte suspensions}

The increase in $[\mathrm{NaCl}]$ from $10^{-5} \mathrm{M}-10^{-1} \mathrm{M}$ causes the aggregation and morphological changes of GO nanosheets directing to the formation of large clusters (Fig. 9 (a)). Qualitatively, the aggregate sizes are the largest in GO-30 suspension as compared to the aggregate sizes in GO-60 and GO-120 suspensions. Moreover, independent of the ultrasonication time, the addition of $10^{-5} \mathrm{M}-10^{-1} \mathrm{M}\left[\mathrm{MgCl}_{2}\right]$ causes an increase in the aggregation of GO nanosheets, as shown in Fig. 9 (b). The zeta potential of the GO nanosheets decreases in the presence of $\mathrm{NaCl}$ and $\mathrm{MgCl}_{2}$, as shown in section $\mathrm{S}_{7}$ in the supporting information. The zeta potential for the GO-30, GO-60, and GO-120 decreases from $-49.1 \mathrm{mV}$ to $-7.1 \mathrm{mV},-47.4 \mathrm{mV}$ to $-8.3 \mathrm{mV}$, and $-46.4 \mathrm{mV}$ to $-9.9 \mathrm{mV}$ respectively in the presence of $10^{-1} \mathrm{M}[\mathrm{NaCl}]$. Similarly, the zeta potential for these samples decreases from $-49.1 \mathrm{mV}$ to $-1.8 \mathrm{mV},-47.4 \mathrm{mV}$ to $-1.9 \mathrm{mV}$ and $-46.4 \mathrm{mV}$ to $-2.3 \mathrm{mV}$ in the presence of $10^{-1}$ $\mathrm{M}\left[\mathrm{MgCl}_{2}\right]$. It is evident by the zeta potential that the addition of electrolytes shields the negative charge on GO surface, which further results in the subsequent reduction in the electrostatic repulsion and the formation of flocs or aggregates of GO in aqueous dispersion. The zeta potential is measured for $0.02 \mathrm{mg} / \mathrm{ml}$ of GO nanosheets, and thus the introduction of $10^{-1} \mathrm{M}[\mathrm{NaCl}]$ or $\mathrm{MgCl}_{2}$ leads to sedimentation of $\mathrm{GO}$ nanosheets over time. In contrast, the suspensions in this study are prepared with $25 \mathrm{mg} / \mathrm{ml}$ of $\mathrm{GO}$, and in the presence of $10^{-1} \mathrm{M}$ $[\mathrm{NaCl}]$ or $\left[\mathrm{MgCl}_{2}\right]$, the aggregation of $\mathrm{GO}$ nanosheets does not lead to the sedimentation but instead leads to the formation of solid like gels.

In agreement with these results, our microscopy results qualitatively show that the size of GO aggregates decreases with the increase in ultrasonication time. Moreover, the aggregates appear to be larger for $\mathrm{GO}-\mathrm{MgCl}_{2}$ suspensions than corresponding $\mathrm{GO}-\mathrm{NaCl}$ suspensions. The dynamic light scattering (DLS) measurements (section $\mathrm{S}_{8}$ in supporting information) confirm the qualitative microscopy images. The effective diameter or the aggregate size continually increases with the electrolyte concentration more prominently with the $\mathrm{MgCl}_{2}$ than $\mathrm{NaCl}$. 
Literature studies report that monovalent cations facilitate the aggregation between the GO nanosheets by promoting face-to-face aggregation and the effective shielding of the negative charge on GO sheets, but the bivalent cation $\mathrm{Mg}^{2+}$ not only destabilizes the GO by suppressing the electrical double layer (EDL) but also induces microstructural changes and promotes the transformation of GO structure into 1D, 2D and 3D nanomaterials based on the initial size of $\mathrm{GO}$ nanosheets. Thus, $\mathrm{Mg}^{2+}$ cations are also able to form stable cross-linked networks or "bridges" between the GO sheets with the help of - $\mathrm{COOH}$ groups available at GO nanosheets edges[39,40]. The increased ultrasonication time leads to a small increase in the d-spacing with $8.15 \AA, 8.20 \AA, 8.27 \AA$ for GO-30, GO-60 and GO-120 respectively. The intercalation of cations is reported to increase the layer-to-layer distance or d-spacing of GO nanosheets. The intercalation of $\mathrm{Mg}^{2+}$ ions between the $\mathrm{GO}$ layers reflects in the $\mathrm{d}$-spacing of $8.92 \AA$ in GO-30 with $10^{-1} \mathrm{M}\left[\mathrm{MgCl}_{2}\right]$. The $\mathrm{Mg}^{2+}$ ionic radius is approximately $0.72 \AA$, which gives a clear indication that the $\mathrm{Mg}^{2+}$ ions are indeed intercalated between the layers of GO in GO-30 and similar observations have also been reported in the literature earlier[41,42]. The d-spacing are $8.89 \AA$ and $8.43 \AA$ in GO-60 and GO-120 dispersions with $10^{-1} \mathrm{M}\left[\mathrm{MgCl}_{2}\right]$ suggesting significant intercalation and coordination of $\mathrm{Mg}^{2+}$ in GO-60 but not in GO-120. The effective $\mathrm{Mg}^{2+}$ coordination with the $-\mathrm{COOH}$ group GO-30 and GO-120 is also discussed in detail in in the XPS analysis below in Fig.10. On the other hand, no significant increase in d-spacing of GO-30, GO-60 and GO-120 has been observed when $\mathrm{Na}^{+}$cations are introduced into the system as mentioned in section $S_{9}$.

Thus, we hypothesize that independent of ultrasonication time, due to the $\mathrm{Mg}^{2+}$ mediated aggregation of GO nanosheets, the $\mathrm{G}_{\mathrm{p}}^{\prime}$ of $\mathrm{GO}-\mathrm{MgCl}_{2}$ suspensions is higher than GO-NaCl suspensions. 

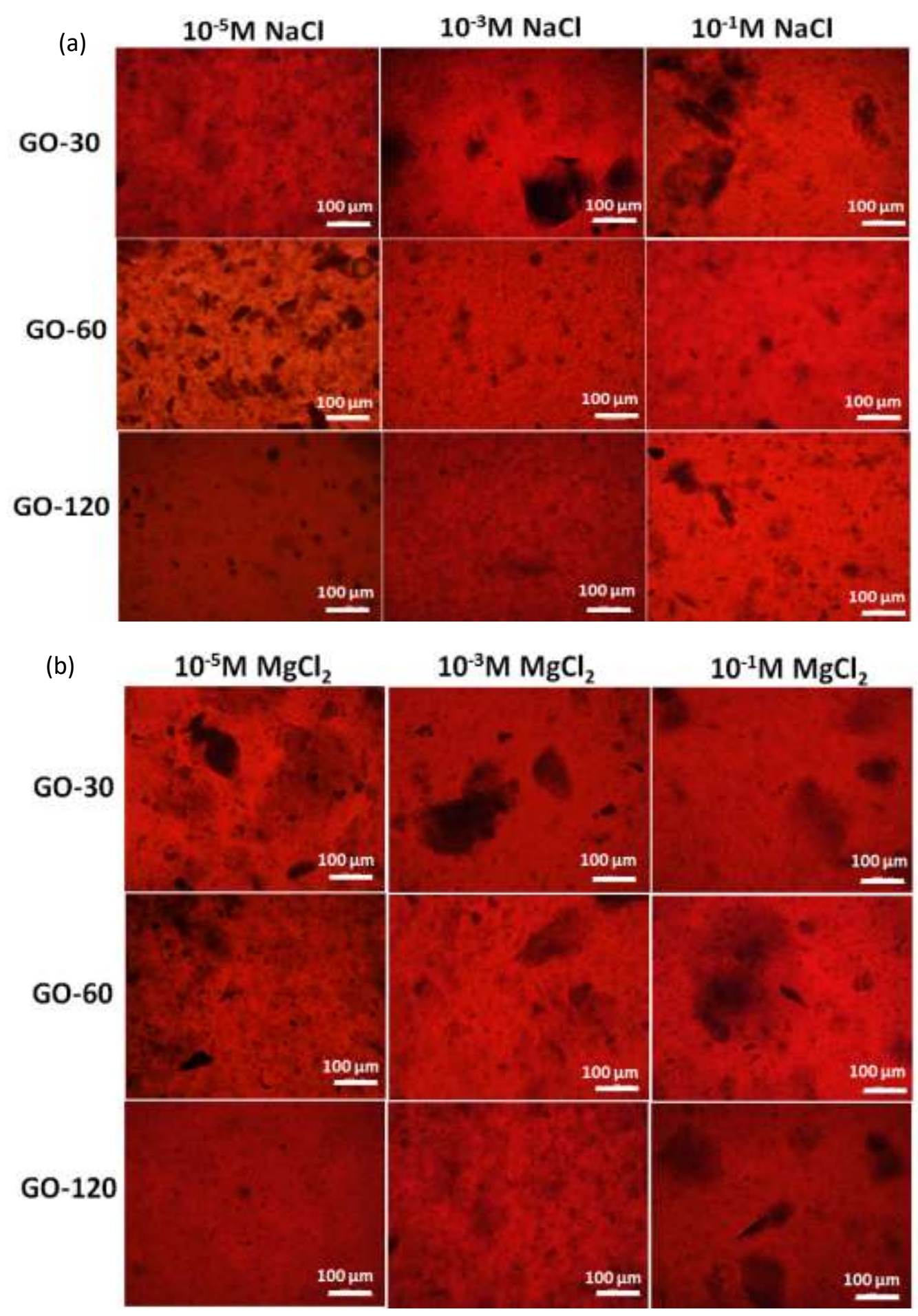

Fig. 9. Optical microscopy images of GO-electrolyte suspensions at different (a) $[\mathrm{NaCl}]$ and (b) $\left[\mathrm{MgCl}_{2}\right]$

The $\mathrm{Mg}^{2+}$ mediated interactions are also reflected in the core level XPS spectra related to the $\mathrm{C} 1 \mathrm{~s}$ region of lyophilized GO and GO-electrolyte gels as represented by Fig. 10 and section $\mathrm{S}_{10}$ of supporting information. The binding energies of $\mathrm{C}=\mathrm{C}$ and $\mathrm{C}-\mathrm{OH}$ bonds are assigned as $284.6 \mathrm{eV}$ and $285.6 \mathrm{eV}$, respectively. Further, the peaks at $286.7 \mathrm{eV}, 288.2 \mathrm{eV}$ 
and $289.4 \mathrm{eV}$ are assigned to the $-\mathrm{C}=\mathrm{O} / \mathrm{C}-\mathrm{O}-\mathrm{C}, \mathrm{C}=\mathrm{O}$ and $-\mathrm{C}(\mathrm{O}) \mathrm{O}$ functional groups respectively. The $\mathrm{C}-\mathrm{Cl}$ bond peak around $286.5 \mathrm{eV}$ completely coincides with the $-\mathrm{C}=\mathrm{O}$ peak. As a result of $\mathrm{MgCl}_{2}$ addition in GO-30, the intensity of the epoxy/ether groups situated at $286.7 \mathrm{eV}$ reduces significantly whereas the $\mathrm{C}-\mathrm{OH}$ peak intensity increases due to the increased component of hydroxyl/alkoxide $\mathrm{C}$ suggesting the possible formation of alcohol groups as a result of epoxide group's ring opening mechanism[43]. Interestingly, this phenomenon is observed more prominently in the case of GO-30 with $\mathrm{MgCl}_{2}$ as compared to GO-120 with $\mathrm{MgCl}_{2}$ (Fig. 10 (b, e)). On the other hand, no such increase is visible when $\mathrm{NaCl}$ is added in either GO-30 or GO-120 samples (Fig. 10 (c), (f)).

Also, the C1s component related to carboxyl acid groups shifts slightly from $289.4 \mathrm{eV}$ to $289.1 \mathrm{eV}$ and $289.4 \mathrm{eV}$ to $289.3 \mathrm{eV}$ for GO-30 and GO-120 respectively suggesting the effective coordination between $\mathrm{Mg}^{2+}$ and carboxyl acid groups on the edges (Fig. 10 (b, e)). The shift in the $-\mathrm{C}(\mathrm{O}) \mathrm{O}$ peak is again predominant in the case of $\mathrm{GO}-30$ with $\mathrm{MgCl}_{2}$. On the other hand, no significant change has been observed in the XPS spectrum of GO after the addition of monovalent cation $\mathrm{Na}^{+}$using $\mathrm{NaCl}$ electrolyte. The chemical interactions of nonultrasonicated and 120 min. ultrasonicated $\mathrm{GO}$ with $\mathrm{NaCl}$ and $\mathrm{MgCl}_{2}$ are also evident by FTIR as shown by section $\mathrm{S}_{11}$ of the supporting information.

Considering $\mathrm{C} \mathrm{sp}{ }^{2}$ peak at $284.6 \mathrm{eV}$ as an internal reference, the $\mathrm{Mg} 2 \mathrm{p}$ binding energy peak obtained around $50.6 \mathrm{eV}$ (as shown in section $\mathrm{S}_{10}$ (b) of supporting information) can be attributed to the ionic bond of $\mathrm{Mg}$ and $\mathrm{O}$. Therefore, it can be inferred from the above observations that a potential interaction mechanism between the $\mathrm{GO}$ and $\mathrm{Mg}^{2+}$ is the formation of $\mathrm{Mg}-\mathrm{O}$ bond serving as a cross-linker between adjacent $\mathrm{GO}$ nanosheets. On the other hand, as compared to GO-30, relatively smaller Mg 2p peaks are observed for GO-60 and GO-120 with $\mathrm{MgCl}_{2}$, suggesting relatively fewer $\mathrm{Mg}-\mathrm{O}$ bonds between the neighboring GO nanosheets. Interestingly, our experimental observations are in line with the observations reported by Lin et. al in case of GO papers doped with $\mathrm{Mg}^{2+}$ and $\mathrm{Cl}^{-}[42]$.

Therefore, consistent with the literature, the XPS spectrum suggests that GO nanosheets interact with $\mathrm{Mg}^{2+}$ ions in two different modes. The strongest mode of binding occurs when $\mathrm{Mg}^{2+}$ binds with carboxylic acid groups at the edges of individual GO nanosheet, causing the edge-to-edge bridging of $\mathrm{GO}$ nanosheets. In addition, $\mathrm{Mg}^{2+}$ can also induce the ring-opening of epoxides to create $\mathrm{C}-\mathrm{OH}$ groups that interact with already present carbonyl groups causing inter-layer crosslinking of GO nanosheets. Similar results have also been reported in the case of GO papers by Lin et al. and Park et al [41,42]. 

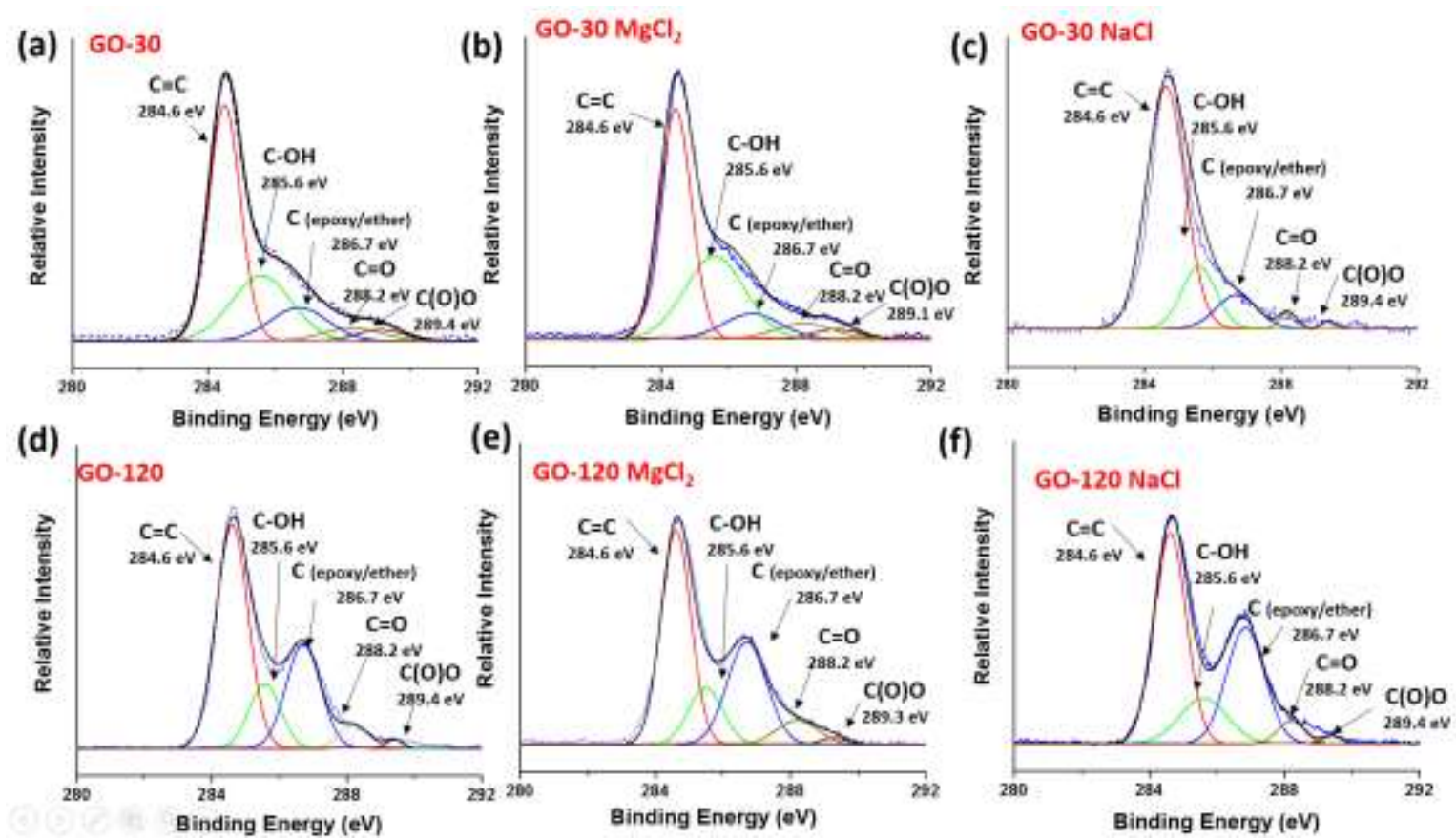

Fig. 10 Deconvoluted core level XPS spectra of C1s region for GO nanosheets after (a-c) 30 minutes ultrasonication and addition of $10^{-1} \mathrm{M}\left[\mathrm{MgCl}_{2}\right],[\mathrm{NaCl}]$ (d-f) 120 minutes ultrasonication and addition of $10^{-1} \mathrm{M}\left[\mathrm{MgCl}_{2}\right],[\mathrm{NaCl}]$

We believe that with an increase in ultrasonication time, the extent of $\mathrm{Mg}^{2+}$ bridging the nanosheets decreases due to the formation of new un-functionalized nanosheet edges, resulting in the reduced $\mathrm{G}_{\mathrm{p}}^{\prime}$ of gels comprising of smaller $\mathrm{GO}$ nanosheets. Therefore, $\mathrm{G}_{\mathrm{p}}$ for GO- $\mathrm{MgCl}_{2}$ suspensions with the largest GO sheets (GO-30) is the highest among all the suspensions analyzed.

\subsection{Kinetics of Adsorption of MB by lyophilized GO-30, GO-60 and GO-120 suspensions and their gel-like derivatives}

The MB adsorption experiments are performed up to $60 \mathrm{hrs}$. with lyophilized GO-30, GO-60, GO-120, and the corresponding GO-electrolyte gels. The visual pictures of MB adsorption are shown in section $S_{12}$ of supporting information. The adsorption kinetics curve showing the adsorption capacity of different adsorbents is shown in Fig. 11. Lyophilized GO-30-10 $\mathrm{M}[\mathrm{NaCl}]$ and $\mathrm{GO}-30-10^{-1} \mathrm{M}\left[\mathrm{MgCl}_{2}\right]$ gels show the maximum adsorption capacity of 319.5 $\mathrm{mg} / \mathrm{g}$ and $324.5 \mathrm{mg} / \mathrm{g}$ respectively. A slight increase in the adsorption capacity is 
demonstrated by lyophilized GO-60-10-1 $\mathrm{M}[\mathrm{NaCl}]$ and GO-60-10-1 $\mathrm{M}\left[\mathrm{MgCl}_{2}\right]$ gels with the maximum adsorption capacity of $330.4 \mathrm{mg} / \mathrm{g}$ and $333.1 \mathrm{mg} / \mathrm{g}$, respectively. The lowest dye adsorption capacities of $249.2 \mathrm{mg} / \mathrm{g}$ and $262.7 \mathrm{mg} / \mathrm{g}$ are obtained for both $\mathrm{GO}-120-10^{-1} \mathrm{M}$ $[\mathrm{NaCl}]$ and $\mathrm{GO}-120-10^{-1} \mathrm{M}\left[\mathrm{MgCl}_{2}\right]$ based adsorbents.
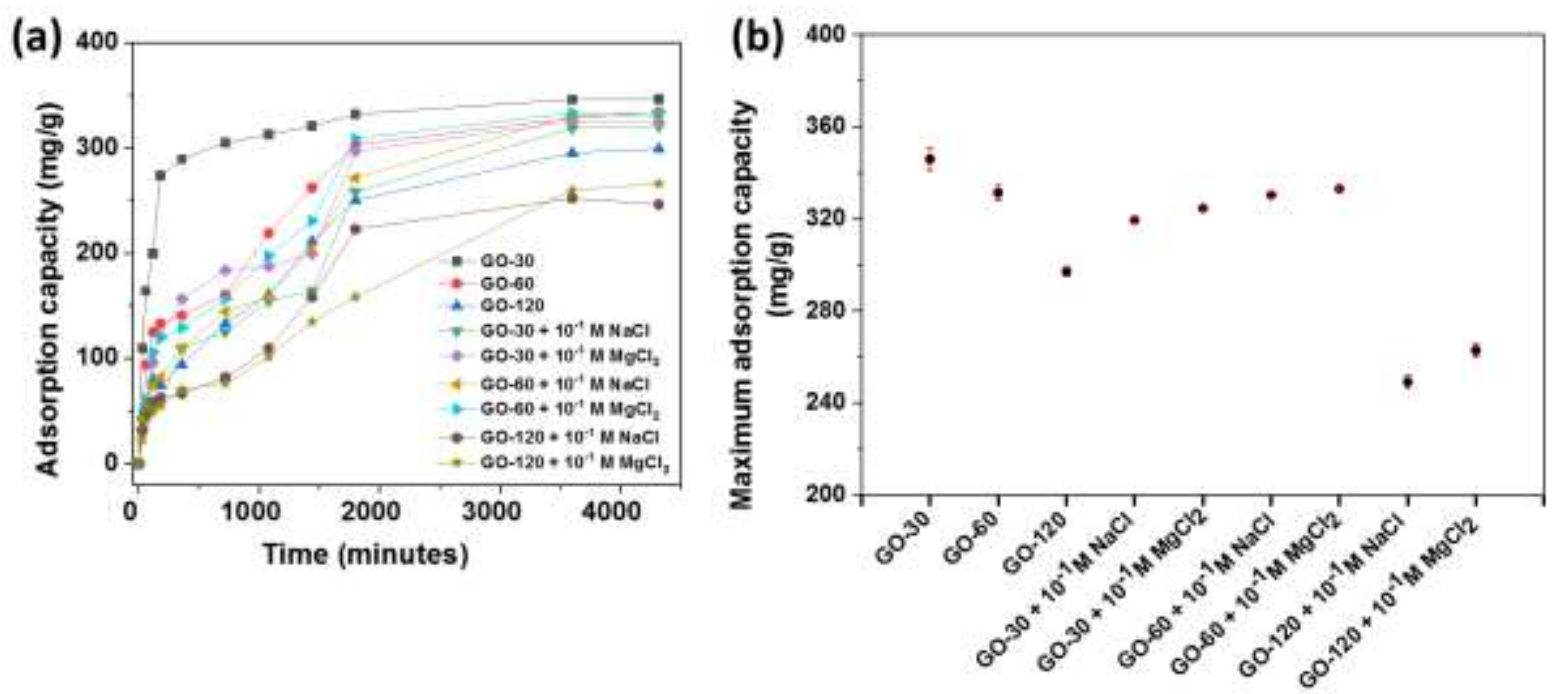

Fig. 11. (a) Adsorption capacity (b) maximum adsorption capacity for lyophilized GO and GO-electrolyte gels with different sized GO nanosheets (the errors are of the size of symbols)

The morphology obtained by introducing $10^{-1} \mathrm{M}[\mathrm{NaCl}]$ and $\left[\mathrm{MgCl}_{2}\right]$ is studied by SEM and the obtained micrographs are shown by Fig.12. The adjecent GO nanosheets tend to join each other as a result of shielded negative charge on their surfaces by cations as well as due to the cation mediated cross-linking at the nanosheet edges. These interactions give rise to interaggregate pores between the nanosheet clusters as well as pores due to the cross-linking of nanosheets. It can be inferred from the SEM micrographs that the interaggregate pores created by GO-30 with both the electrolytes interactions are less in number but larger in size and volume (Fig.12 (a, c)). The larger size of nanosheets and pores also in turn contributes in creating more inter-space and a greater extent of active sites. These features make the GO-30 based gel structures superior adsorbents for MB. Therefore, the adsorption efficiency of such gels is found to be higher in our studies. On the other hand, the size reduction of GO in case of GO-120 leads to the crowding of nanosheets resulting in increased compactness in the gellike structure and low site exposure as shown in Fig.12 (b, d). Another plausible reason might be the removal of oxy-functional groups during the size reduction process as well as 
strong $\pi-\pi$ interactions as mentioned by Shen et al [23]. Due to these features, the adsorption efficiency of GO-120 based adsorbents (gels) decreases sufficiently in our studies as compared to GO-30 and GO-60 based adsorbents.

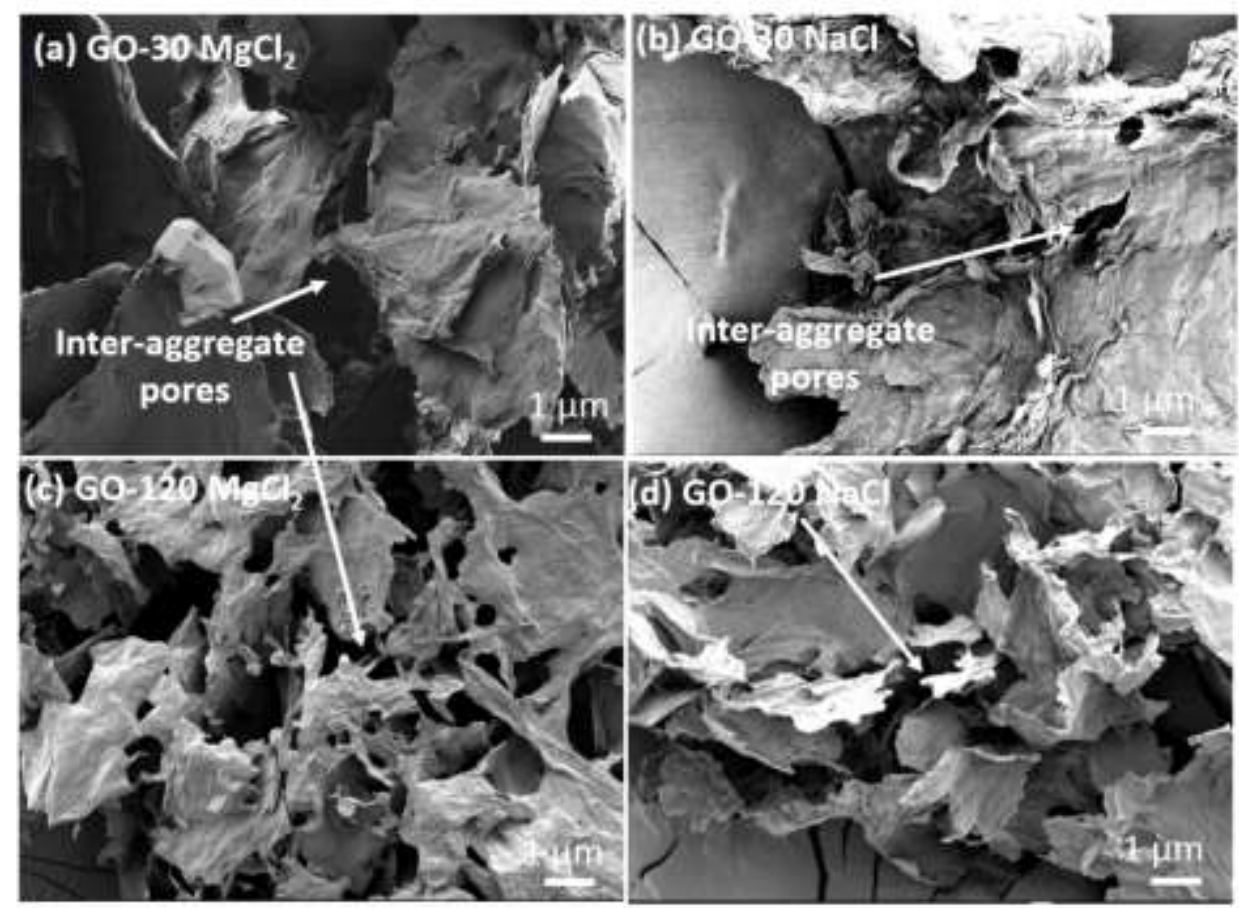

Fig. 12. SEM micrographs of GO-electrolytes samples (lyophilized) with different size of GO nanosheets (GO30 and GO-120) prepared with $(\mathrm{a}, \mathrm{c}) 10^{-1} \mathrm{M}$ of $\left[\mathrm{MgCl}_{2}\right]$ and $(\mathrm{b}, \mathrm{d}) 10^{-1} \mathrm{M}[\mathrm{NaCl}]$

To investigate the leached electrolyte concentration in the aqueous media, the $\left[\mathrm{Mg}^{2+}\right]$ and $\left[\mathrm{Na}^{+}\right]$are measured in DI water using ICP-OES on pre-defined time intervals. The maximum possible $\left[\mathrm{Mg}^{2+}\right]$ and $\left[\mathrm{Na}^{+}\right]$are calculated as $13.40 \mathrm{ppm}$ and $18.62 \mathrm{ppm}$ respectively, when $10^{-1}$ $M$ of each electrolyte is dispersed in water without GO. The leached $\left[\mathrm{Mg}^{2+}\right]$ and $\left[\mathrm{Na}^{+}\right]$are 6.93, $3.6 \mathrm{ppm}$ for the lyophilized GO-30-10-1 $\mathrm{M}\left[\mathrm{MgCl}_{2}\right]$ and GO-30-10-1 $\mathrm{M}[\mathrm{NaCl}]$ gels respectively. Similarly, for GO-60-10-1 $\mathrm{M}\left[\mathrm{MgCl}_{2}\right]$ and $\mathrm{GO}-60-10^{-1} \mathrm{M}[\mathrm{NaCl}]$ lyophilized gels, the leached $\left[\mathrm{Mg}^{2+}\right]$ and $\left[\mathrm{Na}^{+}\right]$ions is around $1.11 \mathrm{ppm}$ and $6.91 \mathrm{ppm}$, respectively. Interestingly, for the GO-120-10-1 $\mathrm{M}\left[\mathrm{MgCl}_{2}\right]$ and $\mathrm{GO}-120-10^{-1} \mathrm{M}[\mathrm{NaCl}]$, the leached ionic concentration is 12.3 and $15.92 \mathrm{ppm}$, respectively that suggests that the gel structure is not able to withhold the electrolyte in itself and it comes out in water immediately. However, in all the cases, the overall leached $\left[\mathrm{Mg}^{2+}\right]$ and $\left[\mathrm{Na}^{+}\right]$ions is quite lower than the maximum permissible limit (30 and $20 \mathrm{ppm}$ respectively) in water resources as recommended by the BIS Indian standards. 


\section{Conclusions}

The ultrasonication time and addition of electrolytes can be an effective strategy to modulate the rheological behavior of GO suspensions. Increasing the time of ultrasonication without any chemical treatment can promote aggregation of GO nanosheets as reflected by the increase in the $\mathrm{G}_{\mathrm{p}}^{\prime}$. The presence of electrolytes $\left(\mathrm{NaCl}, \mathrm{MgCl}_{2}\right)$ increases the elastic moduli for GO aqueous dispersions. Solid gel-like suspensions are formed for 30, 60 and 120 min of ultrasonication in the presence of $10^{-1} \mathrm{M}$ of $\mathrm{NaCl}$ or $\mathrm{MgCl}_{2}$. The maximum $\mathrm{G}_{\mathrm{p}}^{\prime}$ is obtained for the lowest time of ultrasonication $\left(30 \mathrm{~min}\right.$ ) and in the presence of $10^{-1} \mathrm{M} \mathrm{MgCl}_{2}$. The detailed XPS analysis shows that the $\mathrm{Mg}^{2+}$ mediated bridging of GO nanosheets is the primary factor of aggregation and the subsequent large $\mathrm{G}_{\mathrm{p}}^{\prime}$. Moreover, ultrasonicating for 120 min not only causes the reduction in the size of GO nanosheets, but also leads to a reduction in the $\mathrm{Mg}^{2+}$ mediated crosslinking and hence a decrease in $\mathrm{G}_{\mathrm{p}}^{\prime}$. Lyophilized GO-electrolyte gels can also function as adsorbents for MB dye. GO-120-electrolyte gels are the least effective adsorbents for the adsorption of MB dye.

\section{Acknowledgments}

Financial support for this work is provided by Department of Science and Technology, Government of India, SERB (EMR/2016/003840) and IIT Gandhinagar. Abhijeet acknowledges the IIT Gandhinagar Overseas Research Fellowship for funding his stay and research at Institut des NanoSciences de Paris, Sorbonne Université, Paris. The authors also acknowledge the central instrumentation facility (CIF, IITGN) for providing the overall characterization facilities.

\section{References}

[1] A.K. Geim, K.S. Novoselov, The rise of graphene, Nat. Mater. 6 (2007) 183-191. https://doi.org/10.1038/nmat1849.

[2] G. Eda, G. Fanchini, M. Chhowalla, Large-area ultrathin films of reduced graphene oxide as a transparent and flexible electronic material, Nat. Nanotechnol. 3 (2008) 270-274. https://doi.org/10.1038/nnano.2008.83. 
[3] A.K. Geim, G. Opportunities, Graphene : Status and Prospects, (n.d.).

[4] J. Zhao, S. Pei, W. Ren, L. Gao, H.-M. Cheng, Efficient Preparation of Large-Area Graphene Oxide Sheets for Transparent Conductive Films, ACS Nano. 4 (2010) 52455252. https://doi.org/10.1021/nn1015506.

[5] Z. Liu, J.T. Robinson, X. Sun, H. Dai, PEGylated Nanographene Oxide for Delivery of Water-Insoluble Cancer Drugs, J. Am. Chem. Soc. 130 (2008) 10876-10877. https://doi.org/10.1021/ja803688x.

[6] S. Ghosh, W. Bao, D.L. Nika, S. Subrina, E.P. Pokatilov, C.N. Lau, A.A. Balandin, Dimensional crossover of thermal transport in few-layer graphene, Nat. Mater. 9 (2010) 555-558. https://doi.org/10.1038/nmat2753.

[7] A.A. Balandin, D.L. Nika, S. Ghosh, E.P. Pokatilov, Lattice thermal conductivity of graphene flakes: Comparison with bulk graphite, Appl. Phys. Lett. 94 (2009). https://doi.org/10.1063/1.3136860.

[8] M.J. Allen, V.C. Tung, R.B. Kaner, Honeycomb Carbon: A Review of Graphene, Chem. Rev. 110 (2010) 132-145. https://doi.org/10.1021/cr900070d.

[9] X. Dong, P. Wang, W. Fang, C.Y. Su, Y.H. Chen, L.J. Li, W. Huang, P. Chen, Growth of large-sized graphene thin-films by liquid precursor-based chemical vapor deposition under atmospheric pressure, Carbon N. Y. 49 (2011) 3672-3678. https://doi.org/10.1016/j.carbon.2011.04.069.

[10] X. Xu, Z. Zhang, J. Dong, D. Yi, J. Niu, M. Wu, L. Lin, R. Yin, M. Li, J. Zhou, S. Wang, J. Sun, X. Duan, P. Gao, Y. Jiang, X. Wu, H. Peng, R.S. Ruoff, Z. Liu, D. Yu, E. Wang, F. Ding, K. Liu, Ultrafast epitaxial growth of metre-sized single-crystal graphene on industrial $\mathrm{Cu}$ foil, Sci. Bull. 62 (2017) 1074-1080. https://doi.org/10.1016/j.scib.2017.07.005.

[11] L. Niu, M. Li, X. Tao, Z. Xie, X. Zhou, A.P.A. Raju, R.J. Young, Z. Zheng, Saltassisted direct exfoliation of graphite into high-quality, large-size, few-layer graphene sheets, Nanoscale. 5 (2013) 7202-7208. https://doi.org/10.1039/c3nr02173d.

[12] K. Parvez, Z.-S. Wu, R. Li, X. Liu, R. Graf, X. Feng, K. Müllen, Exfoliation of Graphite into Graphene in Aqueous Solutions of Inorganic Salts, J. Am. Chem. Soc. 
136 (2014) 6083-6091. https://doi.org/10.1021/ja5017156.

[13] D.R. Dreyer, S. Park, C.W. Bielawski, R.S. Ruoff, The chemistry of graphene oxide, Chem. Soc. Rev. 39 (2010) 228-240. https://doi.org/10.1039/b917103g.

[14] S. Saxena, T.A. Tyson, E. Negusse, Investigation of the Local Structure of Graphene Oxide, J. Phys. Chem. Lett. 1 (2010) 3433-3437. https://doi.org/10.1021/jz1014339.

[15] D.C. Marcano, D. V. Kosynkin, J.M. Berlin, A. Sinitskii, Z. Sun, A. Slesarev, L.B. Alemany, W. Lu, J.M. Tour, Improved synthesis of graphene oxide, ACS Nano. 4 (2010) 4806-4814. https://doi.org/10.1021/nn1006368.

[16] R. Hummers, W. S.; Offeman, E., Preparation of Graphitic Oxide, J. Am. Chem. Soc. 208 (1957) 1937.

[17] U. Khan, A. O’Neill, H. Porwal, P. May, K. Nawaz, J.N. Coleman, Size selection of dispersed, exfoliated graphene flakes by controlled centrifugation, Carbon N. Y. 50 (2012) 470-475. https://doi.org/10.1016/j.carbon.2011.09.001.

[18] S. Pan, I.A. Aksay, Factors Controlling the Size of Graphene Oxide Sheets Produced via the Graphite Oxide Route, ACS Nano. 5 (2011) 4073-4083. https://doi.org/10.1021/nn200666r.

[19] A. Ojha, P. Thareja, Graphene-based nanostructures for enhanced photocatalytic degradation of industrial dyes, Emergent Mater. (2020) 1-12. https://doi.org/10.1007/s42247-020-00081-6.

[20] K.H. Liao, Y.S. Lin, C.W. MacOsko, C.L. Haynes, Cytotoxicity of graphene oxide and graphene in human erythrocytes and skin fibroblasts, ACS Appl. Mater. Interfaces. 3 (2011) 2607-2615. https://doi.org/10.1021/am200428v.

[21] F. Liu, S. Chung, G. Oh, T.S. Seo, Three-dimensional graphene oxide nanostructure for fast and efficient water-soluble dye removal, ACS Appl. Mater. Interfaces. 4 (2012) 922-927. https://doi.org/10.1021/am201590z.

[22] C. Sage, J. Deng, B. Lei, A. He, X. Zhang, L. Ma, S. Li, C. Zhao, Toward 3D graphene oxide gels based adsorbents for high-efficient water treatment via the promotion of biopolymers, 263 (2013) 467-478. 
[23] Y. Shen, X. Zhu, B. Chen, Size effects of graphene oxide nanosheets on the construction of three-dimensional graphene-based macrostructures as adsorbents, J. Mater. Chem. A. 4 (2016) 12106-12118. https://doi.org/10.1039/c6ta04112d.

[24] F. Perreault, A.F. de Faria, S. Nejati, M. Elimelech, Antimicrobial Properties of Graphene Oxide Nanosheets: Why Size Matters, ACS Nano. 9 (2015) 7226-7236. https://doi.org/10.1021/acsnano.5b02067.

[25] Y. He, C. Tong, L. Geng, L. Liu, C. Lü, Enhanced performance of the sulfonated polyimide proton exchange membranes by graphene oxide: Size effect of graphene oxide, J. Memb. Sci. 458 (2014) 36-46. https://doi.org/10.1016/j.memsci.2014.01.017.

[26] J.E. Kim, T.H. Han, S.H. Lee, J.Y. Kim, C.W. Ahn, J.M. Yun, S.O. Kim, Graphene Oxide Liquid Crystals, Angew. Chemie Int. Ed. 50 (2011) 3043-3047. https://doi.org/10.1002/anie.201004692.

[27] F. Lin, X. Tong, Y. Wang, J. Bao, Z.M. Wang, Graphene oxide liquid crystals: synthesis, phase transition, rheological property, and applications in optoelectronics and display, Nanoscale Res. Lett. 10 (2015) 435. https://doi.org/10.1186/s11671-0151139-1.

[28] B. Dan, N. Behabtu, A. Martinez, J.S. Evans, D. V. Kosynkin, J.M. Tour, M. Pasquali, I.I. Smalyukh, Liquid crystals of aqueous, giant graphene oxide flakes, Soft Matter. 7 (2011) 11154-11159. https://doi.org/10.1039/c1sm06418e.

[29] A. Ojha, P. Thareja, Electrolyte induced rheological modulation of graphene oxide suspensions and its applications in adsorption, Appl. Surf. Sci. 435 (2018) 786-798. https://doi.org/10.1016/j.apsusc.2017.11.157.

[30] B. Konkena, S. Vasudevan, Glass, gel, and liquid crystals: Arrested states of graphene oxide aqueous dispersions, J. Phys. Chem. C. 118 (2014) 21706-21713. https://doi.org/10.1021/jp507266t.

[31] S. Naficy, R. Jalili, S.H. Aboutalebi, R.A. Gorkin, K. Konstantinov, P.C. Innis, G.M. Spinks, P. Poulin, G.G. Wallace, Graphene oxide dispersions: Tuning rheology to enable fabrication, Mater. Horizons. 1 (2014) 326-331. https://doi.org/10.1039/c3mh00144j. 
[32] Y. Xu, H. Bai, G. Lu, C. Li, G. Shi, Flexible Graphene Films via the Filtration of Water-Soluble Noncovalent Functionalized Graphene Sheets, J. Am. Chem. Soc. 130 (2008) 5856-5857. https://doi.org/10.1021/ja800745y.

[33] A. Lerf, H. He, M. Forster, J. Klinowski, Structure of Graphite Oxide Revisited ", J. Phys. Chem. B. 102 (1998) 4477-4482. https://doi.org/10.1021/jp9731821.

[34] N. Koumakis, G. Petekidis, Two step yielding in attractive colloids: Transition from gels to attractive glasses, Soft Matter. 7 (2011) 2456-2470. https://doi.org/10.1039/c0sm00957a.

[35] J.R. Stokes, W.J. Frith, Rheology of gelling and yielding soft matter systems, Soft Matter. 4 (2008) 1133-1140. https://doi.org/10.1039/b719677f.

[36] O.C. Compton, Z. An, K.W. Putz, B.J. Hong, B.G. Hauser, L. Catherine Brinson, S.T. Nguyen, Additive-free hydrogelation of graphene oxide by ultrasonication, Carbon N. Y. 50 (2012) 3399-3406. https://doi.org/10.1016/j.carbon.2012.01.061.

[37] N. V. Medhekar, A. Ramasubramaniam, R.S. Ruoff, V.B. Shenoy, Hydrogen bond networks in graphene oxide composite paper: Structure and mechanical properties, ACS Nano. 4 (2010) 2300-2306. https://doi.org/10.1021/nn901934u.

[38] P. Sollich, F. Lequeux, P. Hébraud, M.E. Cates, Rheology of soft glassy materials, Phys. Rev. Lett. 78 (1997) 2020-2023. https://doi.org/10.1103/PhysRevLett.78.2020.

[39] L. Wu, L. Liu, B. Gao, R. Muñoz-Carpena, M. Zhang, H. Chen, Z. Zhou, H. Wang, Aggregation kinetics of graphene oxides in aqueous solutions: Experiments, mechanisms, and modeling, Langmuir. $29 \quad$ (2013) 15174-15181. https://doi.org/10.1021/la404134x.

[40] K. Yang, B. Chen, X. Zhu, B. Xing, Aggregation, Adsorption, and Morphological Transformation of Graphene Oxide in Aqueous Solutions Containing Different Metal Cations, Environ. Sci. Technol. $50 \quad$ (2016) 11066-11075. https://doi.org/10.1021/acs.est.6b04235.

[41] K. Sungjin Lee, G. Bozoklu, W. Cai, Ḱ.S.T. Nguyen, R.S. Ruoff, Graphene Oxide Papers Modified by Ions, Divalent Mechanical, Enhancing Cross-linking, Prop. Chem. Park. 2 (2014). 
[42] X. Lin, X. Shen, X. Sun, X. Liu, Y. Wu, Z. Wang, J.K. Kim, Graphene Oxide Papers Simultaneously Doped with $\mathrm{Mg} 2+$ and Cl- for Exceptional Mechanical, Electrical, and Dielectric Properties, ACS Appl. Mater. Interfaces. 8 (2016) 2360-2371. https://doi.org/10.1021/acsami.5b11486.

[43] MOULDER, J. F., Handbook of X-Ray Photoelectron Spectroscopy, Phys. Electron. (1995) 230-232. 
Supporting information

\title{
Nanosheet size-dependent rheology, microstructure, adsorption properties of graphene oxide-electrolyte dispersions and adsorbents
}

\author{
Abhijeet Ojha ${ }^{1}$, Helen Ibrahim ${ }^{2,3}$, Natalia Alyabyeva ${ }^{2}$, Rémi Lazzari ${ }^{2}$, Michel Goldmann ${ }^{2,4}$ \\ and Prachi Thareja ${ }^{5^{*}}$ \\ ${ }^{1}$ Biological Engineering, Indian Institute of Technology, Gandhinagar, Gujarat, India - 382355 \\ ${ }^{2}$ Sorbonne Université, CNRS-UMR 7588, Institut des NanoSciences de Paris, 75005, Paris \\ ${ }^{3}$ LICORNE, ECE Paris Ecole d'Ingénieurs, Immeuble POLLUX, Paris, France -75015 \\ ${ }^{4}$ Synchrotron SOLEIL L'Orme des Merisiers, Saint Aubin, Gif-sur-Yvette, France-91192 \\ ${ }^{5}$ Chemical Engineering, Indian Institute of Technology, Gandhinagar, Gujarat, India- 382355
}

Corresponding author:prachi@iitgn.ac.in

\section{$\mathrm{S}_{1 .}$ (a) Synthesis of graphene oxide (GO) by Hummer's process}

Briefly, in Hummer's method, graphite powder $(5.0 \mathrm{gm})$ is first entirely dissolved in concentrated $\mathrm{H}_{2} \mathrm{SO}_{4}, 115 \mathrm{ml}$ in a $1000 \mathrm{ml}$ round bottom flask at room temperature using a magnetic stirrer. $\mathrm{NaNO}_{3}, 2.5 \mathrm{gm}$ is then added to the solution and stirred vigorously for 30 minutes, and the flask is kept in an ice bath. $\mathrm{KMnO}_{4}, 15.0 \mathrm{gm}$ is then added slowly while maintaining the temperature of the reaction flask below $30^{\circ} \mathrm{C}$, this exothermic reaction results in a dark green colored suspension. Further, the ice bath is replaced with a water bath maintained at $35^{\circ} \mathrm{C}$, and the solution is stirred for 3 hours forming a highly viscous and dark brown coloured suspension. Now the reaction flask is shifted again to the ice bath. To ensure that the temperature is below $30^{\circ} \mathrm{C}, \mathrm{r} 230 \mathrm{ml}$ ultra-pure deionized (DI) water $(18.2 \mathrm{M} \Omega \mathrm{cm}$ resistivity) is slowly added drop-wise into the reaction. The resulting suspension is muddycolored which is mixed into $700 \mathrm{ml}$ of deionized (DI) water followed by quenching with $\sim 12$ ml $\mathrm{H}_{2} \mathrm{O}_{2}$ to eliminate unreacted $\mathrm{KMnO}_{4}$. The quenching results in a stable light yellow graphite oxide suspension.

To get rid of the remaining impurities, the above suspension is centrifuged at around $7600 \mathrm{rpm}$ following decantation and further re-suspension in $\mathrm{HCl}(\sim 4 \%)$ and $\mathrm{DI}$ water respectively. Subsequently, to prepare aqueous GO nanosheet suspension, the graphite oxide suspension is re-dispersed into DI water and sonicated with an ultrasonicator (Sonic 
Vibracell-VC-505, $500 \mathrm{~W}, 20 \mathrm{kHz}$, amplitude 35\%, $10 \mathrm{sec}$. on/off pulse, $1 \mathrm{hr}$. ON time). The settled un-exfoliated graphite oxide is removed by high speed centrifugation for 3-5 minutes. Finally, aqueous GO dispersion is dialyzed by placing it inside a $35 \mathrm{~mm}$ dialysis tube made up of cellulose membrane and immersed in DI water, which is replaced repeatedly upto $40 \mathrm{hrs}$. This GO suspension is termed as "as-prepared" GO suspension in our experiments.

$\mathrm{S}_{1}$. (b) The volume fraction of GO is calculated by using the formula -

$$
\phi_{G O}=\phi_{m}\left(\left[1-\phi_{m}\right] \frac{\rho_{\mathrm{GO}}}{\rho_{\mathrm{water}}}+\phi_{m}\right)^{-1}
$$

Where, $\phi_{\mathrm{m}}=\mathrm{GO}$ weight fraction, $\rho_{\mathrm{GO}}=1.32 \mathrm{~g} / \mathrm{ml}$ and $\rho_{\text {water }}=1 \mathrm{~g} / \mathrm{ml}$

$\mathrm{S}_{2}$. The standard UV absorption curve for Methylene Blue (MB)

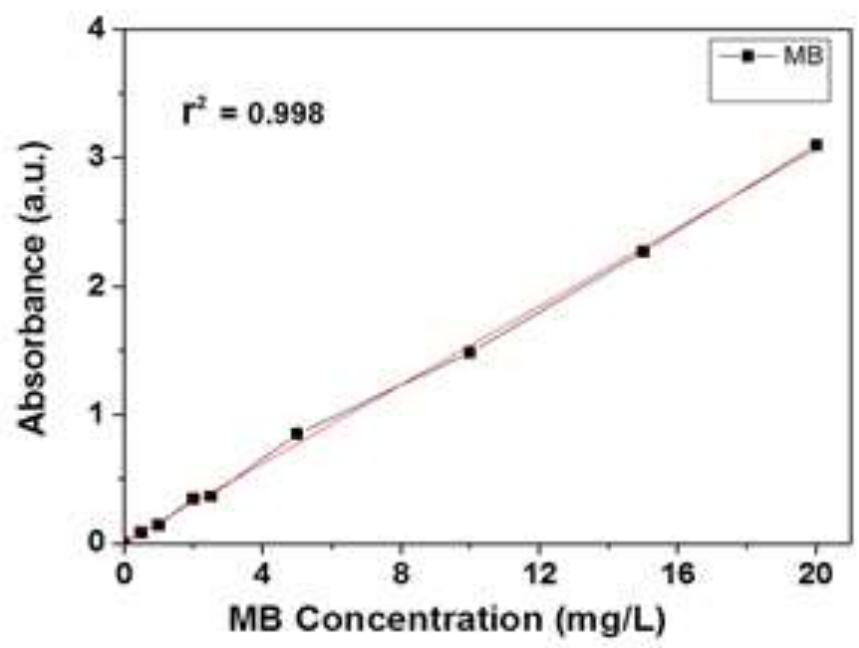

Fig. The standard UV absorption curve for cationic dye methylene blue (MB) measured at $665 \mathrm{~nm}$ 
$\mathrm{S}_{3}$. Rheological studies with electrolyte free GO suspensions after various sonication times

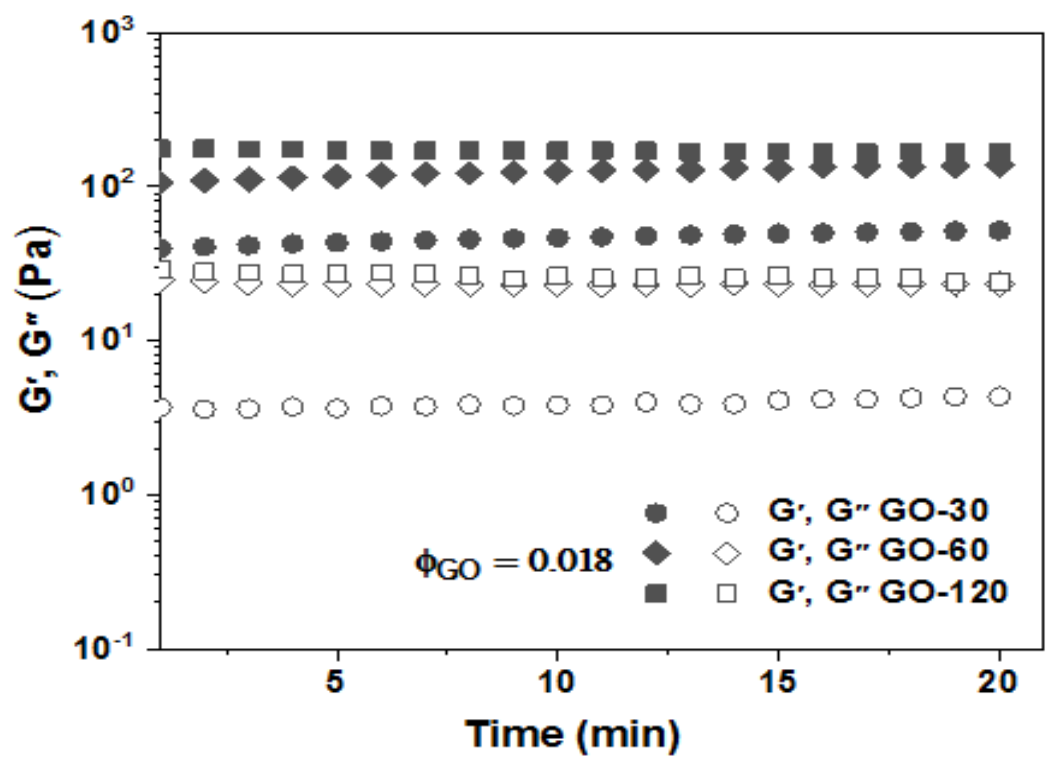

Fig. Oscillatory time sweep experiment with $\mathrm{GO}$ aqueous dispersion (at $\phi_{\mathrm{GO}}=0.018$ ) ultra-sonicated for different time intervals as 30 minutes, 60 minutes and 120 minutes respectively

$\mathrm{S}_{4}$ Rheological studies of suspensions prepared by GO-30, GO-60 and GO-120 with different concentrations of $\mathrm{NaCl}$

(a)

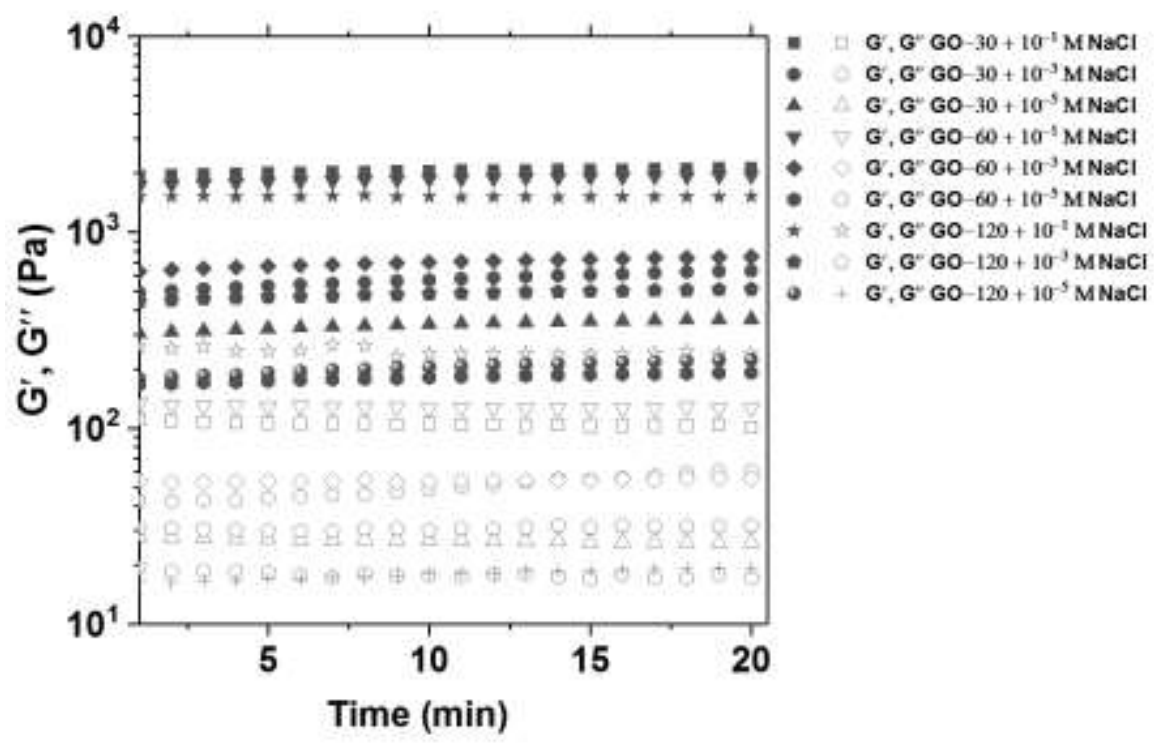


(b)

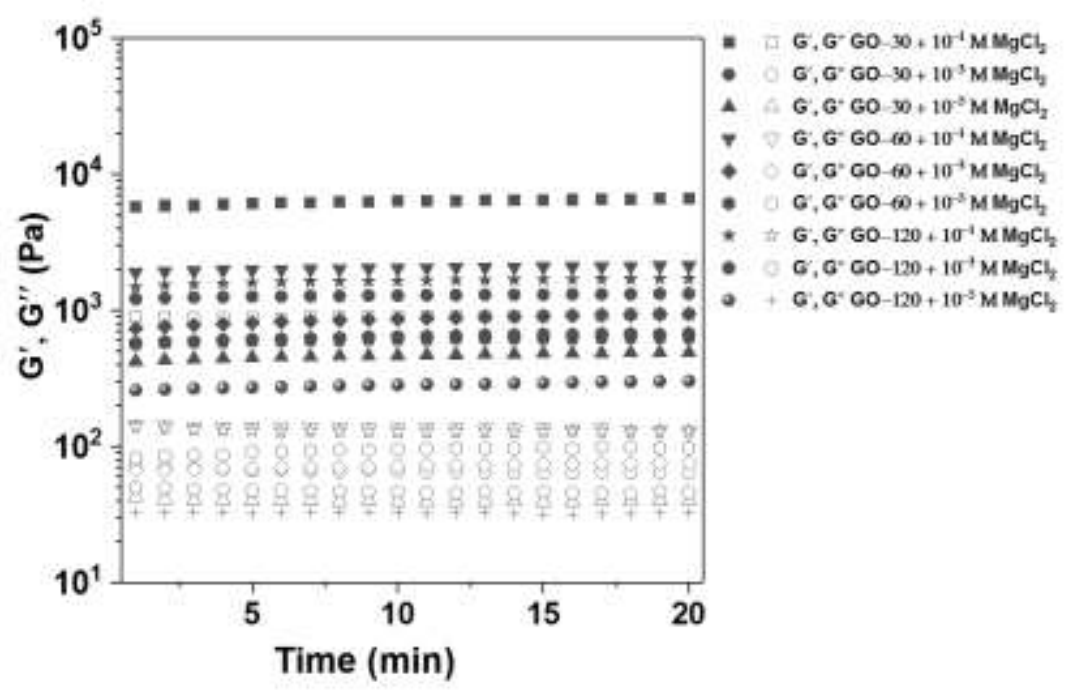

Fig. Oscillatory time sweep experiment with $\mathrm{GO}$ aqueous dispersion (at $\phi_{\mathrm{GO}}=0.018$ ) ultra-sonicated for different time intervals (30,60,120 minutes) and mixed with electrolyte solutions as (a) $\mathrm{NaCl}$ and (b) $\mathrm{MgCl}_{2}$

$\mathrm{S}_{5}$. Large Amplitude Oscillatory strain sweep (LAOS) experiments
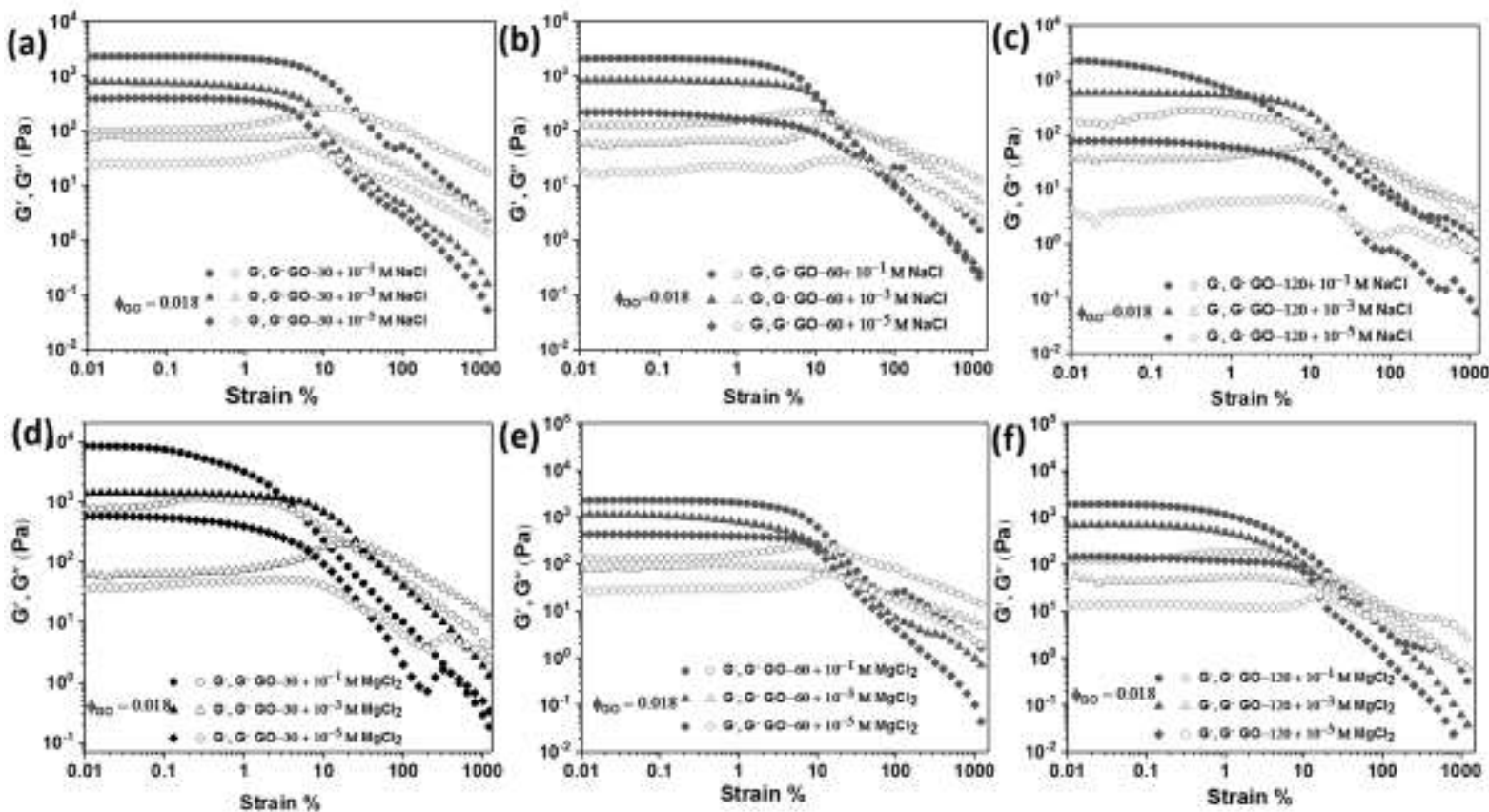

Fig. Large amplitude oscillatory strain sweep experiments of GO-30, GO-60, and GO-120 suspensions with varying $[\mathrm{NaCl}]$ and $\left[\mathrm{MgCl}_{2}\right]$ 
$\mathrm{S}_{6}$. Two yield points in Large Amplitude Oscillatory strain sweep experiments (LAOS) experiments
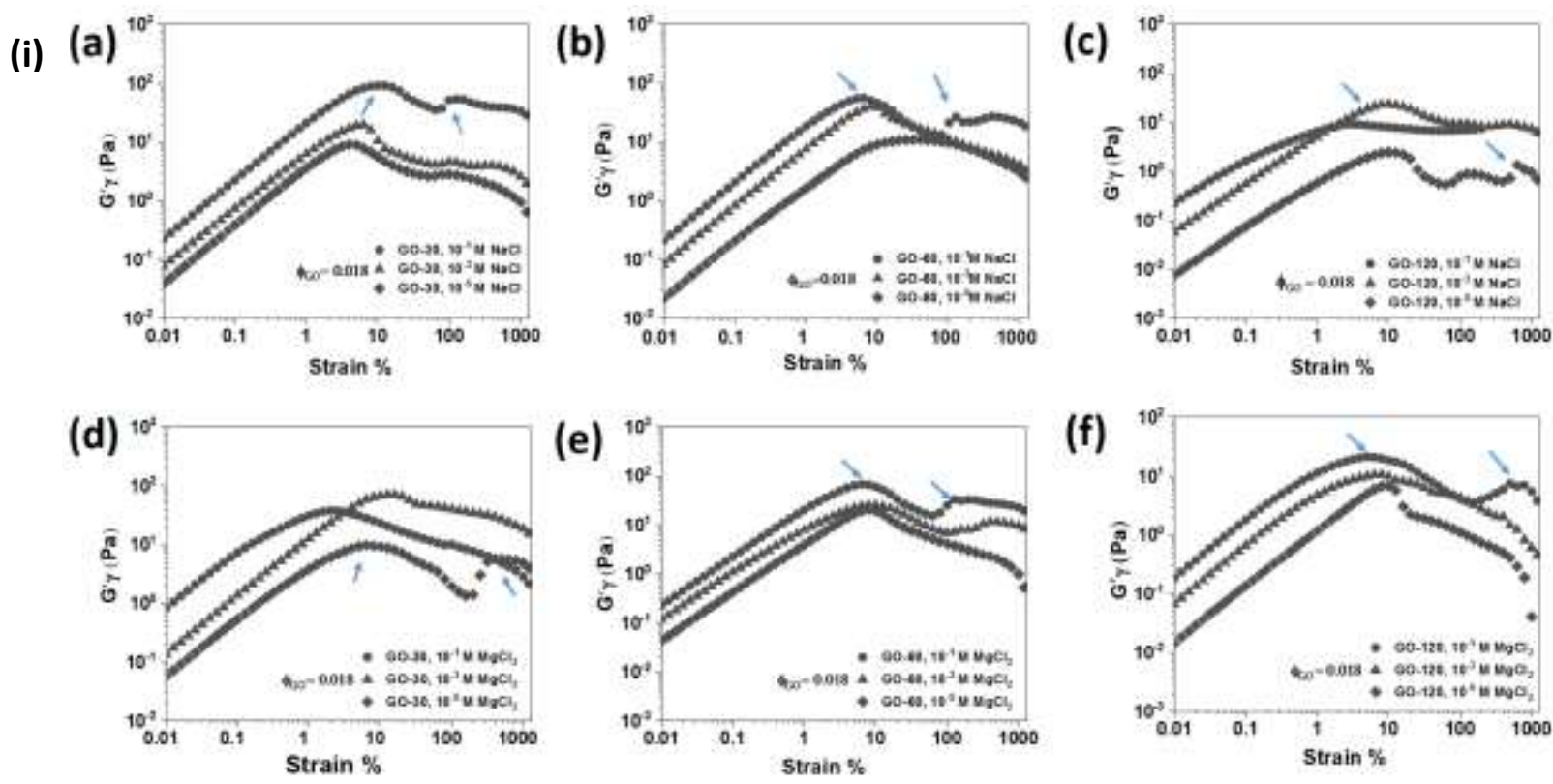

Fig. $\mathrm{G}^{\prime} \gamma$ as a function of applied $\gamma \%$ of GO-electrolyte suspensions at different $[\mathrm{NaCl}]$ and $\left[\mathrm{MgCl}_{2}\right]$

(ii)

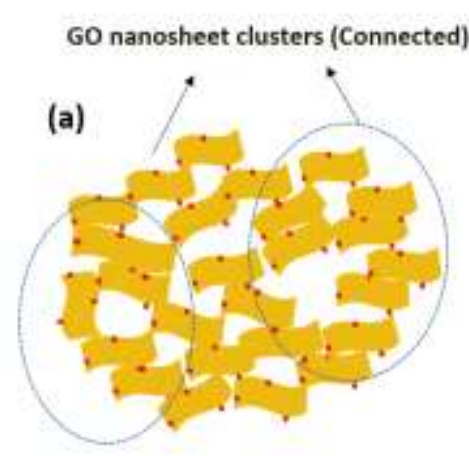

Cross-linked networks of GO (b)

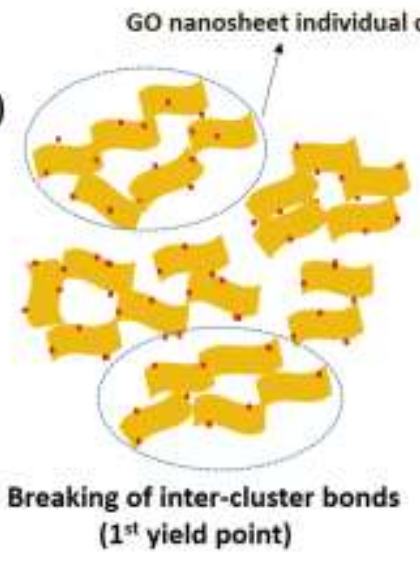

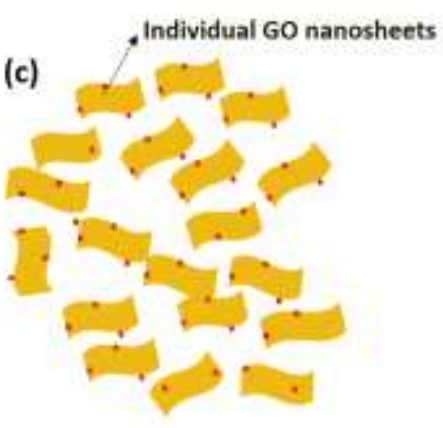

Breaking of inter-particle bonds ( $2^{\text {ed }}$ yield point)

Fig. A schematic representing the breakage of inter-cluster and inter-particle bonds corresponding the two "Yield-points" 
$\mathrm{S}_{7 .}$ Zeta potential analysis

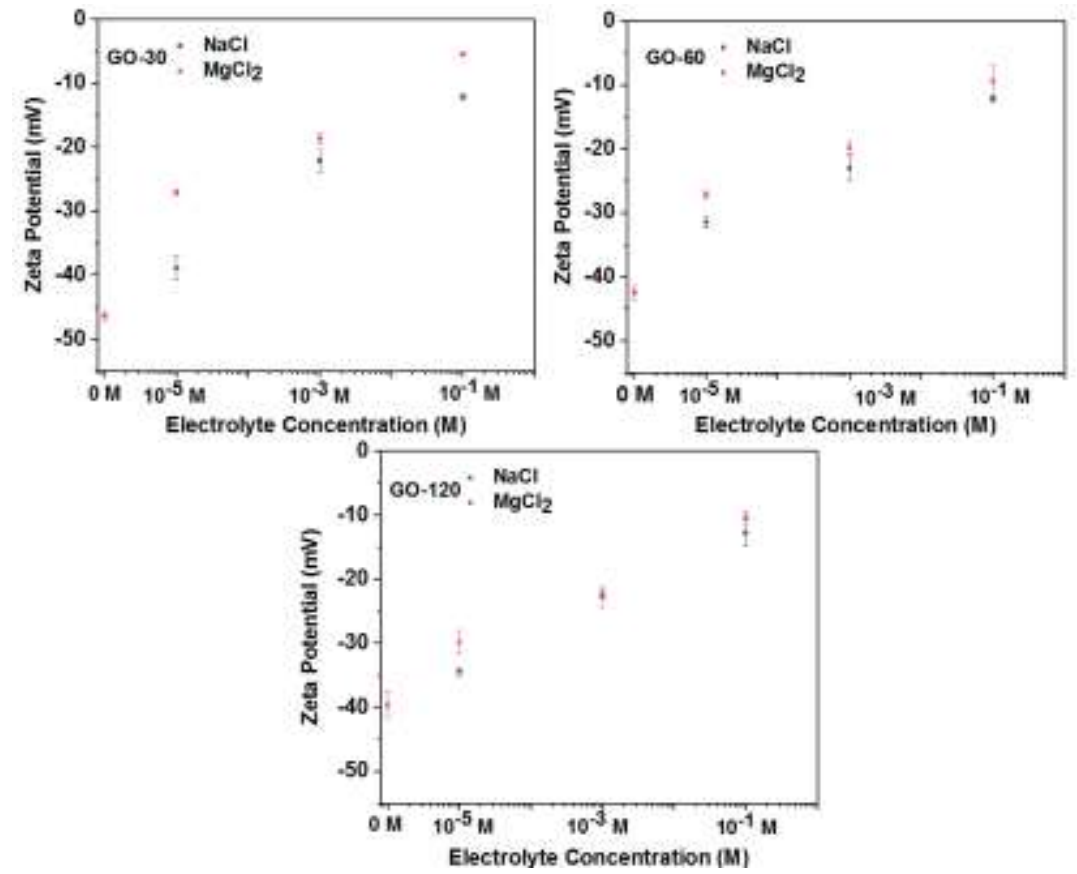

Fig. Zeta potential measurements on GO nanosheets surface after ultra-sonication for variable times and addition of variable concentrations of $\mathrm{NaCl}$ and $\mathrm{MgCl}_{2}$ electrolytes

$\mathrm{S}_{8}$. Dynamic Light Scattering measurements (DLS)

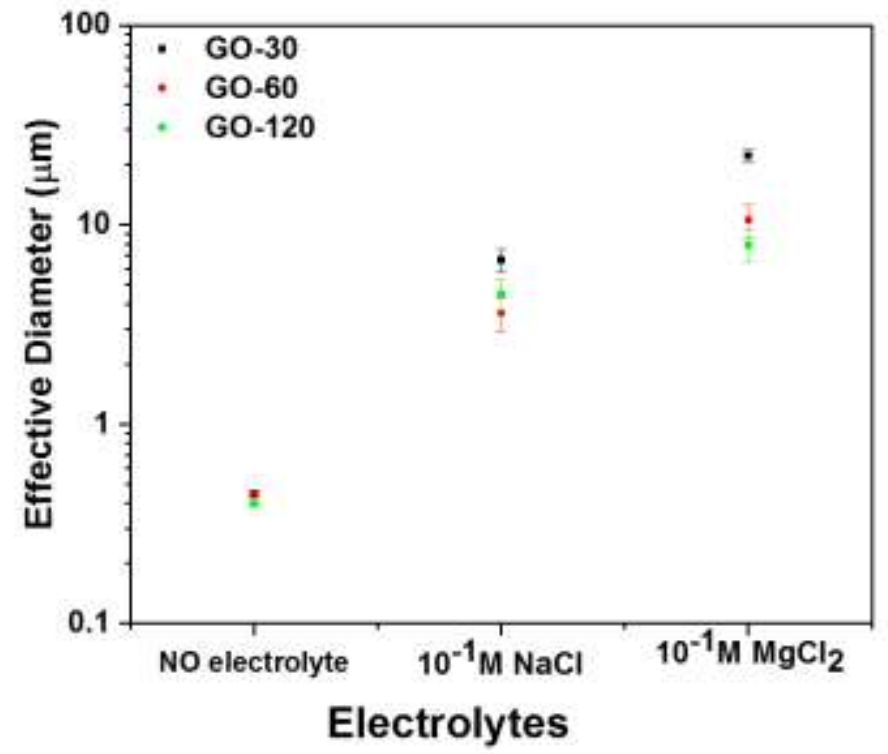

Fig. The particle size measurements of GO nanosheets by DLS in presence of $10^{-1} \mathrm{M}\left[\mathrm{MgCl}_{2}\right]$ and $[\mathrm{NaCl}]$ 
$\mathrm{S}_{9}$. The d-spacing values for GO after ultra-sonication for different time intervals and introduction of different electrolytes for the concerning diffraction peaks

\begin{tabular}{|c|c|c|c|c|}
\hline Sample & $\mathbf{2 \theta}$ & $\begin{array}{c}\text { d-spacing values } \\
\text { (normal condition) }\end{array}$ & $\begin{array}{c}\text { d-spacing values } \\
\left(\text { in } 10^{-1} \mathrm{M} \mathrm{NaCl}\right)\end{array}$ & $\begin{array}{c}\text { d-spacing values } \\
\left(\text { in } 10^{-1} \mathrm{M} \mathrm{MgCl}_{2}\right)\end{array}$ \\
\hline GO-30 & $10.8^{\mathbf{0}}$ & $8.15 \AA$ & $8.20 \AA$ & $8.92 \AA$ \\
\hline GO-60 & $10.67^{\circ}$ & $8.20 \AA$ & $8.22 \AA$ & $8.89 \AA$ \\
\hline GO-120 & $10.60^{\circ}$ & $8.27 \AA$ & $8.28 \AA$ & $8.43 \AA$ \\
\hline
\end{tabular}

$\mathrm{S}_{10}$. XPS spectrum of GO 60 and electrolytes

(a)
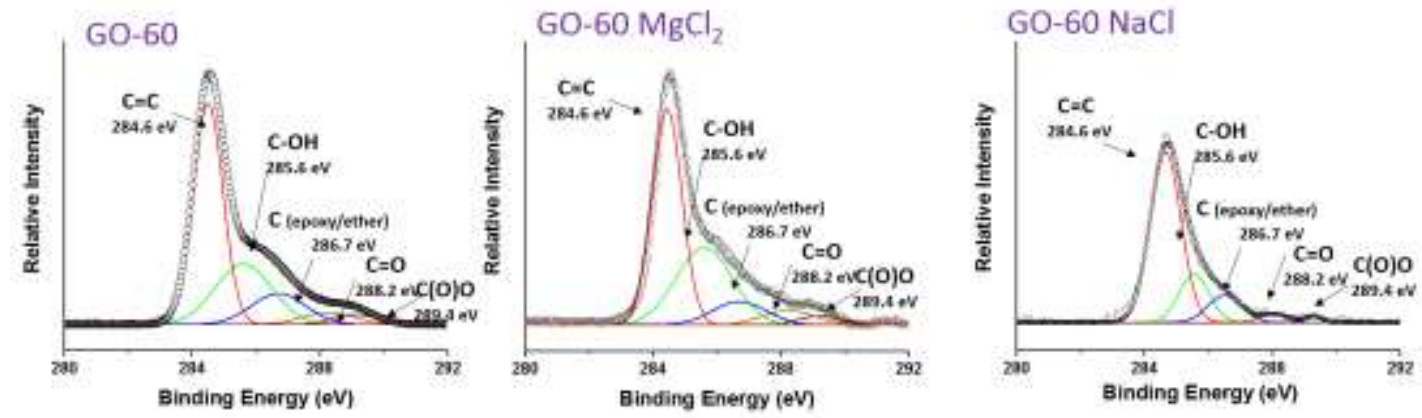

(b)
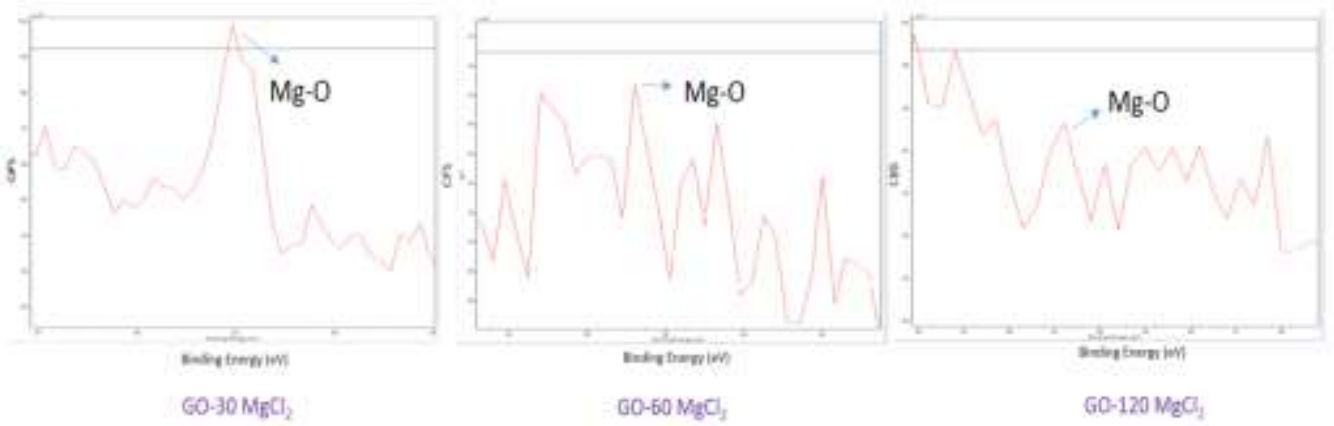

Fig. (a) The Deconvoluted XPS spectra of GO-60 (bare) and GO-60 in presence of $\left.10^{-1} \mathrm{M}^{2} \mathrm{Mgcl}_{2}\right]$ and $[\mathrm{NaCl}]$

(b) The characteristic $\mathrm{Mg} 2 \mathrm{p}$ peak at $50.6 \mathrm{eV}$ in XPS survey spectra for $10^{-1} \mathrm{M} \mathrm{MgCl}_{2}$ and GO sheets ultrasonicated for 30, 60 and 120 minutes respectively 
$\mathrm{S}_{11}$. FTIR absorbance spectra for GO (as prepared ) and GO (with electrolytes)
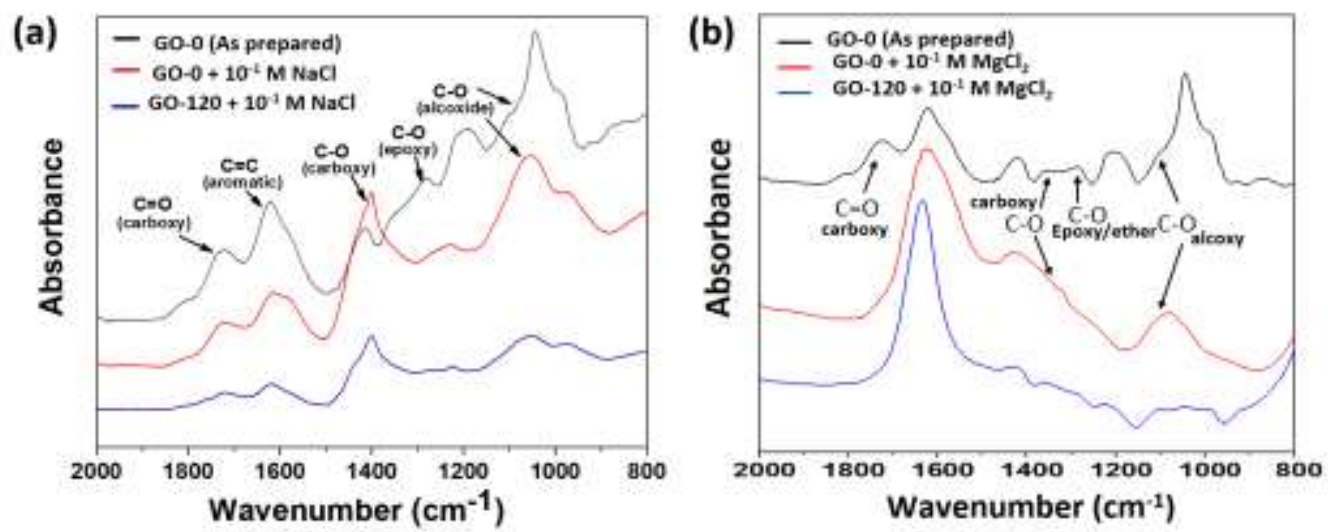

Fig. FTIR spectra showing the absorption peaks of lyophilized GO (as-prepared) and GO-electrolyte suspensions containing GO nanosheets after 0 and 120 minutes' ultra-sonication and addition of $10^{-1} \mathrm{M}[\mathrm{NaCl}]$ and $\left[\mathrm{MgCl}_{2}\right]$ $\mathrm{S}_{12}$. Methylene blue (MB) adsorption study by lyophilized graphene oxide (GO) prepared by variable ultra-sonication time and lyophilized GO-electrolyte suspensions with $10^{-1} \mathrm{M}$ concentration of $[\mathrm{NaCl}]$ and $\left[\mathrm{MgCl}_{2}\right]$

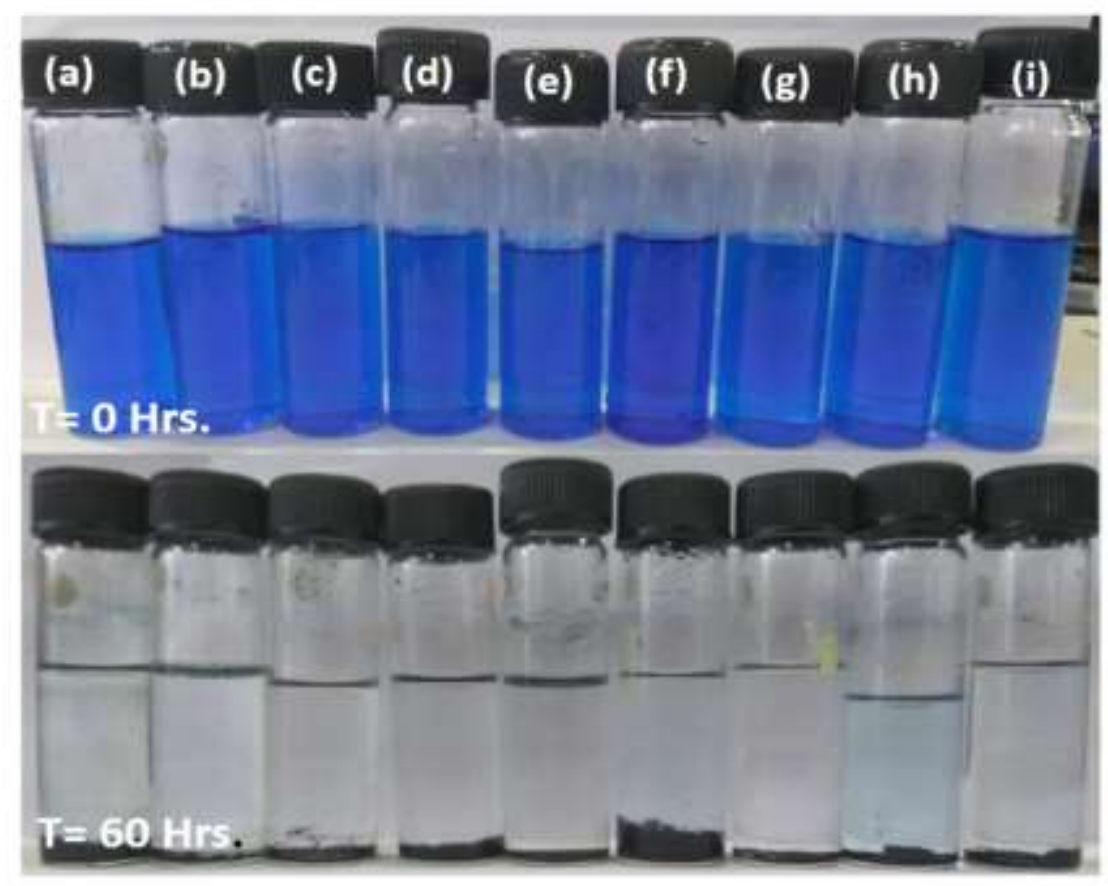

Fig. Visual images of the dye adsorption experiment on $\mathrm{T}=0$ and $\mathrm{T}=60 \mathrm{hrs}$. At $\mathrm{T}=0$, various adsorbents as (a) GO-30 (b) GO-60 (C) GO-120 (d) GO (30)-10 $0^{-1} \mathrm{M} \mathrm{NaCl}$ (e) GO (30)-10-1 $\mathrm{M} \mathrm{MgCl}_{2}$ (f) GO (60)-10-1 $\mathrm{M} \mathrm{NaCl}$ (g) $\mathrm{GO}(60)-10^{-1} \mathrm{M} \mathrm{MgCl}_{2}$ (h) GO (120)-10 $0^{-1} \mathrm{M} \mathrm{NaCl}$ (i) GO (120)-10 $0^{-1} \mathrm{M} \mathrm{MgCl}_{2}$ 